ITC $4 / 49$

Information Technology and Control

Vol. 49 / No. 4 / 2020

pp. 464-481

DOI 10.5755/j01.itc.49.4.2592'7
Leakage-Resilient Outsourced Revocable Certificateless Signature with a Cloud Revocation Server

\begin{tabular}{|l|l}
\hline Received 2020/04/25 & Accepted after revision 2020/10/13 \\
\hline
\end{tabular}

HOW TO CITE: Tseng, Y.-M., Wu, J.-D., Huang, S. S., Tsai, T. T. (2020). Leakage-Resilient Outsourced Revocable Certificateless Signature with a Cloud Revocation Server. Information Technology and Control, 49(4), 464-481. https://doi.org/10.5755/j01.itc.49.4.25927

\title{
Leakage-Resilient Outsourced Revocable Certificateless Signature with a Cloud Revocation Server
}

\author{
Yuh-Min Tseng, Jui-Di Wu, Sen-Shan Huang and Tung-Tso Tsai \\ Department of Mathematics, National Changhua University of Education, Changhua 500, Taiwan
}

Corresponding author: ymtseng@cc.ncue.edu.tw

Certificateless public-key system (CL-PKS) is a significant public-key cryptography and it solves both the key escrow and certificate management problems. Outsourced revocable certificateless public-key system (ORCL-PKS) with a cloud revocation server (CRS) not only provides a revocation mechanism, but also further outsources the revocation functionality to the CRS to reduce the computational burden of the key generation center (KGC). Recently, side-channel attacks have threatened some existing conventional cryptography (including CL-PKS). Indeed, adversaries can apply side-channel attacks to derive fractional constituents of private (or secret) keys to damage the security of these cryptographic protocols (or schemes). To withstand such attacks, leakage-resilient cryptography is an attractive approach. However, little research concerns with leakage-resilient certificateless cryptography. In this paper, the first leakage-resilient outsourced revocable certificateless signature (LR-ORCLS) scheme is presented. The proposed scheme allows adversaries to continually derive fractional constituents of private (or secret) keys and possesses overall unbounded leakage property. In the generic bilinear group (GBG) model, our scheme is shown to be existential unforgeable against adversaries. Finally, the comparisons between the proposed scheme and the previous revocable certificateless signature schemes are provided to demonstrate the merits of the proposed scheme.

KEYWORDS: Signature, Certificateless signature, Revocation, Side-channel attacks, Leakage-resilience. 


\section{Introduction}

Certificateless public-key system (CL-PKS) [1] is a significant public-key cryptography. A CL-PKS setting includes two kinds of participants, namely, users and a key generation center (KGC). The KGC first applies the identity information of a user to derive her/ his identity key, while the user also selects a secret key and sets the associated public key. Hence, the user's private key consists of two components, namely, identity key and self-selected secret key. Since the $\mathrm{KGC}$ is unable to know self-selected secret keys of users, the CL-PKS avoids both the key escrow problem in ID-based public-key systems (ID-PKS) $[4,18]$ and the certificate management in traditional public-key systems $[8,16]$.

In a public-key system, how to revoke compromised users from the system is an essential issue. In some circumstances, users' public keys have to be revoked before their expirations. The certificate revocation list (CRL) [11] is a well-known revocation method in traditional public-key systems. However, this method cannot be applied to both ID-PKS and CL-PKS settings because they do not employ the usage of certificates. Based on the revocation idea in [25], two revocable certificateless encryption schemes [19, 23] were proposed. In 2014, Sun et al. [21] presented a revocable certificateless signature (RCLS) scheme in the random oracle model. To enhance the security, Tsai et al. [24] proposed a new RCLS scheme in the standard model. In 2016, Hung et al. [12] presented a revocable certificateless short signature (RCLSS) scheme. In the RCLSS scheme, the signature size is only a group element. In all RCLS and RCLSS schemes mentioned above, the KGC is responsible for performing the revocation functionality. Recently, Du et al. [7] constructed an outsourced RCLS (ORCLS) scheme with a cloud revocation server (CRS). In the ORCLS scheme, the revocation functionality is outsourced to the CRS to reduce the computational burden of the KGC.

Recently, conventional cryptography has suffered from a new type of attack, called "side-channel attacks", such as timing attack $[5,14]$ and power analysis [15]. Adversaries can apply side-channel attacks to derive fractional constituent of a user's secret (or private) key to damage the security of conventional cryptography. To withstand such attacks, leakage-re- silient cryptography is an attractive approach. Up to now, little research has benn concerned with leakage-resilient certificateless public-key cryptography. In the paper, our aim is to design the first leakage-resilient ORCLS (LR-ORCLS) scheme.

\subsection{The Concept of Leakage-Resilient Cryptography}

Let us introduce the concept of leakage-resilient cryptography here. Indeed, a cryptographic scheme typically includes several computational algorithms. Meanwhile, an adversary can apply side-channel attacks to derive fractional constituent of private (or secret) keys used in each computational algorithm. For representing leakage information, let $f$ and $f(\pi)$, respectively, be a leakage function and its output, where $\pi$ denotes the function input, such as private (or secret) keys. The bit length of the output $f(\pi)$ in each computational algorithm is bounded to a security parameter $\lambda$. For leakage-resilient cryptography, there are two leakage models, namely, bounded leakage model and continual leakage model. For the bounded leakage model $[2,13]$, the overall leakage bit sizes of private (or secret) keys in a cryptographic scheme is restricted during the life cycle. However, the restriction is unpractical. On the other hand, the most accredited model is the continual leakage model that permits adversaries complete leakage-invocated abilities and possesses overall unbounded leakage property $[9,28,29,30]$. In the continual leakage model, there are four properties as indicated below:

- Only computation leakage: An adversary is only permitted to derive fractional constituent of private (or secret) keys involved in the computational algorithm.

- Bounded leakage of single computational algorithm: In each computational algorithm, the bit size of the leakage function output $f(\varpi)$ is bounded to a security parameter $\lambda$.

- Independent leakage: Fractional constituents of private (or secret) keys in any two computational algorithms are mutually independent. To realize the property, the private (or secret) keys must be updated after/before running each computational algorithm. 
- Overall unbounded leakage: By the independent leakage property, the total bit size of leakage information is unbounded.

In the continual leakage model, let us first recall several previous leakage-resilient signature schemes based on traditional public-key, ID-PKS and CLPKS settings. Galindo and Vivek [9] presented a leakage-resilient signature (LRS) scheme based on traditional public-key settings. Galindo and Vivek's scheme owns overall unbounded leakage property and its security is proved in the generic bilinear group (GBG) model [3]. For improving the computational performance of Galindo and Vivek's scheme, Tang et al. [22] then presented a modified LRS scheme. In ID-PKS settings, the first leakage-resilient ID-based signature was presented by Wu et al. [26]. In their scheme, adversaries are permitted to continually derive fractional constituent of private (or secret) keys. Moreover, Wu et al. proved that their scheme is existentially unforgeable against ID and adaptive chosen-message attacks in the GBG model. In CL-PKS settings, based on Xiong et al.'s leakage-resilient certificateless public-key encryption scheme [31], Zhou et al. [32] presented a leakage-resilient certificateless signcryption scheme under the bounded leakage model. In 2018, Wu et al. [27] defined a new adversary model of leakage-resilient CLS (LR-CLS) schemes by adding several key leakage queries under the continual leakage model. Meanwhile, Wu et al. [27] also presented the first LR-CLS scheme which was proved to be secure against adversaries in the new adversary model.

\subsection{Contributions and Organization}

Until now, no leakage-resilient RCLS (LR-RCLS) or ORCLS (LR-ORCLS) scheme withstanding side-channel attacks has been proposed. Indeed, a LR-RCLS scheme can be derived from a LR-ORCLS scheme because the KGC is responsible to play the roles of both the KGC and the CRS in the LR-ORCLS scheme. In the meantime, a LR-ORCLS scheme is better than a LR-RCLS scheme because the revocation functionality is outsourced to the CRS to reduce the computational burden of the KGC. Hence, we will aim at the design of the first LR-ORCLS scheme.

As mentioned earlier, several certificateless cryptographic schemes were proposed, namely, leakage-resilient certificateless signcryption (LR-CLSE) scheme scheme [31], leakage-resilient certificateless signcryption (LR-CLSE) scheme [32] and leakage-resilient certificateless signature (LR-CLS) scheme [27]. Table 1 lists the comparisons between the previous certificateless cryptographic schemes [31, 32, 27] and our LR-ORCLS scheme in terms of cryptographic functionality, overall leakage property, outsourced functionality and revocation functionality. Indeed, these certificateless cryptographic schemes [31, 32, 27 ] did not address the revocation problem. Indeed, in a public-key system, how to revoke compromised users from the system is an essential issue because the compromised users' public keys have to be revoked before their expirations [23, 25].

In this article, the first LR-ORCLS scheme is proposed. We first define the syntax of LR-ORCLS

Table 1

Comparisons between previous certificateless cryptographic schemes and our scheme

\begin{tabular}{|c|c|c|c|c|}
\hline Scheme & $\begin{array}{l}\text { Cryptographic } \\
\text { functionality }\end{array}$ & $\begin{array}{l}\text { Overall leakage } \\
\text { property }\end{array}$ & $\begin{array}{l}\text { Revocation } \\
\text { functionality }\end{array}$ & $\begin{array}{l}\text { Outsourced } \\
\text { functionality }\end{array}$ \\
\hline $\begin{array}{l}\text { Xiong et al.'s LR-CLPKE } \\
\text { scheme [31] }\end{array}$ & Encryption & Bounded & No & No \\
\hline $\begin{array}{l}\text { Zhou et al.'s LR-CLSE } \\
\text { scheme [32] }\end{array}$ & Signcryption & Bounded & No & No \\
\hline $\begin{array}{l}\text { Wu et al.'s LR-CLS scheme } \\
{[27]}\end{array}$ & Signature & Unbounded & No & No \\
\hline Our LR-ORCLS scheme & Signature & Unbounded & Yes & Yes \\
\hline
\end{tabular}


schemes which consists of three participants, namely, a KGC, a CRS and users (signers and verifiers). The $\mathrm{KGC}$ is responsible to generate each user's identity key. At each period, the CRS generates the time update keys of all non-revoked users. Hence, a user's private key consists of three components, namely, identity key, time update key and self-selected secret key. By adding several key leakage queries, we define a new adversary model of LR-ORCLS schemes, which consists of three types of adversaries, namely, Type I (outsider), Type II (revoked user) and Type III (honest-but-curious KGC). In the new adversary model, adversaries are permitted to continually derive fractional constituent of the KGC's master secret key, the CRS's cloud secret key and a signer's secret key used in the associated algorithms. The proposed scheme is shown to be existential unforgeable against Types I, II and III adversaries. Finally, the comparisons between the proposed scheme and the previously related RCLS/ORCLS schemes are provided to demonstrate the merits of the proposed scheme.

The remains of this paper are organized as below. Section 2 demonstrates several preliminaries. The syntax and adversary model of LR-ORCLS schemes are defined in Section 3. In Section 4, a novel LR-ORCLS scheme is proposed. In Section 5, the security of the proposed scheme is formally shown. The comparisons between our scheme and several previous RCLS/ORCLS schemes are given in Section 6. Conclusions and future work are presented in Section 7 .

\section{Preliminaries}

\subsection{Bilinear Groups}

Let $G$ and $\mathrm{G}_{T}$ denote, respectively, an additive group and a multiplicative group of a prime order $p$. Let $P$ be an arbitrary generator of $G$. A bilinear pairing $\hat{e}: G \times G$ $\rightarrow G_{T}$ is an admissible mapping with three properties.

1 Bilinear property: $\hat{e}(a P, b P)=\hat{e}(P, P)^{a b}$, for $a, b \in Z_{q}^{*}$.

2 Non-degenerate property: $\hat{e}(P, P) \neq 1$, which is viewed as a generator of $G_{T}$.

3 Efficient computable property: $\hat{e}(P, Q)$ is computed efficiently, where $Q=c P \in G$ and $c \in Z_{p}^{*}$.

For more detailed definitions of groups, maps and the related parameters, a reader refers to several literatures $[4,17]$.

\subsection{Generic Bilinear Group (GBG) Model and Security Assumption}

By extending the generic group model [20], Boneh et al. [3] presented the generic bilinear group (GBG) model. This model is applied to prove the security of cryptographic schemes/protocols. In this model, if an adversary can find a collision of a group with a large order, it is said that the discrete logarithm problem on the group is resolved.

In the GBG model, there are an additive group $G$ and a multiplicative group $G_{T}$ with the same prime order $p$. In this model, two random injective mappings $\Phi_{G}$ : $Z_{p} \rightarrow \xi_{G}$ and $\Phi_{T}: Z_{p} \rightarrow \xi_{T}$ are, respectively, applied to encode all elements of $G$ and $G_{T}$ to distinct bit-strings. In which, $\xi_{G}$ denotes the set of the encoded bit-strings of $G$ and $\xi_{T}$ is the set of the encoded bit-strings of $G_{T}$. Two sets satisfy $\xi_{G} \cap \xi_{T}=\phi$ and $\left|\xi_{G}\right|=\left|\xi_{T}\right|=p$. In the GBG model, if adversaries want to perform three group operations, they must issue the corresponding public queries $Q_{G}, Q_{T}$ and $Q_{p}$ to a challenger in a security game. Two queries $Q_{G}$ and $Q_{T}$, respectively, denote the addition on $G$ and the multiplication on $G_{T}$. The query $Q_{p}$ denotes the bilinear pairing $\hat{e}$. For $s, t \in Z_{p}^{*}$, three queries satisfy the following properties.

- $Q_{G}\left(\Phi_{G}(s), \Phi_{G}(t)\right) \rightarrow \Phi_{G}(s+t \bmod p)$.

- $Q_{T}\left(\Phi_{T}(s), \Phi_{T}(t)\right) \rightarrow \Phi_{T}(s+t \bmod p)$.

- $Q_{p}\left(\Phi_{G}(s), \Phi_{G}(t)\right) \rightarrow \Phi_{T}(s t \bmod p)$.

Let $P$ be a generator of $G$, we have $P=\Phi_{G}(1)$ and $\hat{e}(P, P)=$ $\Phi_{T}(1)$.

After finishing the security game, if an adversary discovers a collision in $G$ or $G_{T}$, it is said that the discrete logarithm problem on $G$ or $G_{T}$ is resolved. The discrete logarithm assumption is presented as given below.

- Discrete logarithm (DL) assumption: Let $G$ and $\mathrm{G}_{T}$ be an additive group and a multiplicative group of a prime order $p$, respectively. Given a group element $s P \in G$ or $\hat{e}(P, P)^{s} \in G_{T}$ for unknown $s \in Z_{p}^{*}$, no algorithm $A$ with non-negligible probability is able to derive $a$ in polynomial time.

\subsection{The Measure of Leakage Information}

Let us introduce the concept of entropy here. The entropy of a random variable denotes the measure of uncertainty in statistical mechanics. A secret (or private) key can be viewed as a finite random variable. Let $Z$ and $\operatorname{Pr}[Z=z]$ be a finite random variable 
and the associated probability with $Z=z$. In addition, the min-entropy of $Z$ is the predictability value of the largest probability with $Z=z$. The min-entropy and average conditional min-entropy are, respectively, presented as given below.

1 Min-entropy of $Z$ :

$H_{\infty}(Z)=-\log _{2}\left(\max _{Z} \operatorname{Pr}[Z=z]\right)$.

2 Average conditional min-entropy of $Z$ with $E=e$ : $\widetilde{H}_{\infty}(Z \mid E=e)=-\log _{2}\left(E_{e \leftarrow E}[\max \operatorname{Pr}[Z=z \mid E=e]]\right)$.

To address the security influence due to partial leakage of a secret (or private) key, Dodis et al. [6] presented a consequence as indicated below.

Lemma 1. Let $Z$ denote a secret key (i.e. a random variable). Let $\lambda$ be the maximal bit-length of leakage information. Let $h: Z \rightarrow\{0,1\}^{\lambda}$ be a leakage function with input $Z$. Under the event $h(Z)$, the average conditional min-entropy on $Z$ is $\widetilde{H}_{\infty}(Z \mid h(Z)) \geqq H_{\infty}(Z)-\lambda$.

Typically, several secret keys (i.e. multiple random variables) are involved in the computational algorithms of a cryptographic scheme/protocol. To measure the security influence due to partial leakage of polynomials with multiple random variables, Galindo and Vivek [9] presented the following consequences.

Lemma 2. Let $Q_{1}, Q_{2}, \ldots, Q_{n}$ be random variables with probability distributions. Let $F \in Z_{p}\left[Q_{1}, Q_{2}, \ldots, Q_{n}\right]$ denote a polynomial with total degree at most $d$. Let $P_{i}$ denote probability distributions on $Z_{p}$ while $H_{\infty}\left(P_{i}\right)$ $\geqq \log p-\lambda$ holds, where $i=1,2, \ldots, n$ and $0 \leqq \lambda \leqq \log p$. If all $Q_{i}=q_{i} \leftarrow Z_{p}$ with probability distribution $P_{i}$ are independently selected, we have the probability $\operatorname{Pr}\left[F\left(Q_{1}=q_{1}, Q_{2}=q_{2}, \ldots, Q_{n}=q_{n}\right)=0\right] \leqq(d / p) 2^{\lambda}$.

Corollary 1. $\operatorname{Pr}\left[F\left(Q_{1}=q_{1}, Q_{2}=q_{2}, \ldots, Q_{n}=q_{n}\right)=0\right]$ is negligible if $\lambda<(1-\varepsilon) \log p$, where $\varepsilon$ denotes a positive value.

\section{Syntax and Adversary Model}

In this section, the syntax and adversary model of LR-ORCLS schemes are defined.

\subsection{Syntax of LR-ORCLS Schemes}

Let us firstly present the system operation of LR-ORCLS schemes here. In a LR-ORCLS scheme, there are three participants, namely, a key generation center (KGC), a cloud revocation server (CRS) and users (signers and verifiers). The KGC generates identity keys of all users while the CRS generates the time update keys of all non-revoked users at each period $T_{t}$. In addition, each user (signer) chooses a secret key by oneself. Without the loss of generality, a signer with identity $I D$ at period $T_{t}$ wants to sign a message $m s g$. The signer uses his/her identity key, time update key and secret key to generate a signature $\sigma$ and sends the signature tuple (ID, $T_{t}, m s g, \sigma$ ) to a verifier. The system operation of the LR-ORCLS scheme is depicted in Figure 1.

\section{Figure 1}

The system operation of a LR-ORCLS scheme

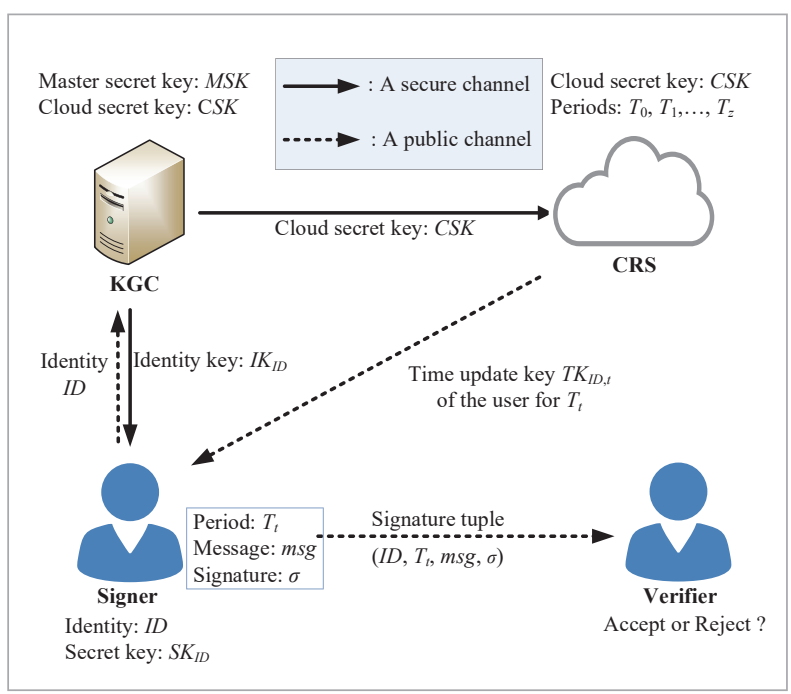

Some notations are summarized below.

- MSK: the KGC's master secret key.

- MPK: the KGC's master public key.

- CSK: the CRS's cloud secret key.

- CPK: the CRS's cloud public key.

- ID: the identity of a user, where $I D \in\{0,1\}^{*}$.

- $S K_{I D}$ : the secret key of the user ID.

- $P K_{I D}$ : the public key of the user $I D$.

- $T_{t}$ : a period $T_{t} \in\{0,1\}^{*}$, for $t=0,1, \ldots, z$, where $z+1$ denotes the amount of periods.

- $I K_{I D}$ : the identity key of the user $I D$.

- $T K_{I D, t}:$ the time update key of the user ID at period $T_{t}$.

- msg: a message.

- $\sigma$ : a signature. 
By extending the syntaxes in [7, 27], the syntax of LR-ORCLS schemes is formally defined as below.

Definition 1. A LR-ORCLS scheme includes eight algorithms:

- Setup: The KGC first sets the master secret key $M S K=\left(M S K_{0,1}, M S K_{0,2}\right)$, the master public key $M P K$, the cloud secret key $C S K=\left(C S K_{0,1}, C S K_{0,2}\right)$ and the cloud public key $C P K$ while securely sending the cloud secret key CSK to the CRS. The KGC sets $z+1$ periods $T_{0}, T_{1}, \ldots, T_{z}$ while publishing public parameters $P P$.

- Identity key extract: For the $i$-th execution of Identity key extract algorithm, the algorithm includes two sub-algorithms IKExtract-1 $\left(M S K_{i-1,1}\right)$ and IKExtract-1 $\left(M S K_{i-1,2}\right)$. Firstly, the $K G C$ sets the new master secret key $\left(M S K_{i, 1}, M S K_{i, 2}\right)$ by using $\left(M S K_{i-1,1}, M S K_{i-1,2}\right)$. By taking as input a user $I D$, the $K G C$ computes the user's identity key $I K_{I D}$ and partial public key $Q_{I D}$. Finally, the $K G C$ securely sends $I K_{I D}$ and $Q_{I D}$ to the user.

- Time update key extract: For the $j$-th execution of Time update key extract algorithm, the algorithm includes two sub-algorithms TKExtract-1 $\left(C S K_{j-1,1}\right)$ and TKExtract-1 $\left(C S K_{j-1,2}\right)$. The CRS sets the new cloud secret key $\left(C S K_{j, 1}, C S K_{j, 2}\right)$ by using $\left(C S K_{j-1,1}\right.$, $C S K_{j-1,2}$ ). By taking a non-revoked user $I D$ and the current period $T_{t}$ as input, the CRS generates and sends the user's time update key $T K_{I D, t}$ and partial public key $R_{I D, t}$ to the user.

- Set secret key: A user ID selects her/his secret key $S K_{I D}=\left(S K_{I D, 0,1}, S K_{I D, 0,2}\right)$ and computes the partial public key $P K_{I D}$.

- Set private key: The private key of a user ID at period $T_{t}$ consists of three components, namely, $I K_{I D}, T K_{I D, t}$ and $S K_{I D}$. The user also sets $I K_{I D}=\left(I K_{I D, 0,1}\right.$, $\left.I K_{I D, 0,2}\right)$ and $S K_{I D}=\left(S K_{I D, 0,1}, S K_{I D, 0,2}\right)$.

- Set public key: Upon receiving the partial public keys $Q_{I D}, R_{I D, t}$ and $P K_{I D}$ of a user $I D$ at period $T_{t}$, the user sets her/his public key tuple $\left(Q_{I D}, R_{I D, t}, P K_{I D}\right)$.

- Signing:For the $k$-th execution of a user ID at period $T_{t}$, this algorithm includes two sub-algorithms Signing-1 (IK $\left.I K_{I D, k-1,1}, T K_{I D, t}, S K_{I D, k-1,1}\right)$ and Signing-2 $\left(I K_{I D, k-1,2}, S K_{I D, k-1,2}\right)$. The signer sets the new identity key $\left(I K_{I D, k, 1}, I K_{I D, k, 2}\right)$ and secret key $\left(S K_{I D, k, 1}, S K_{I D, k, 2}\right)$ by using $\left(I K_{I D, k-1,1} I K_{I D, k-1,2}\right)$ and $\left(S K_{I D, k-1,1}, S K_{I D, k-1,2}\right)$, respectively. By taking a message $m s g$ as input, the user applies $\left(I K_{I D, k, 1}, I K_{I D, k, 2}\right), T K_{I D, t}$ and $\left(S K_{I D, k, 1}\right.$,
$\left.S K_{I D, k, 2}\right)$ to compute a signature $\sigma$. The signature tuple is (ID, $\left.T_{t}, m s g, \sigma\right)$.

- Verifying: By taking (ID, $\left.T_{t}, m s g, \sigma\right)$ as input, a verifier returns either "accept" or "reject".

\subsection{Adversary Model of LR-ORCLS Schemes}

In the continual leakage model, six leakage functions $f_{I K E, i}, h_{I K E, i}, f_{T K E, j,}, h_{T K E, j,}, f_{S I G, k}$ and $h_{S I G, k}$ are applied to model an adversary's capabilities. Namely, the outputs of these leakage functions denote fractional constituents of the private (or secret) keys used in the associated algorithms. Here, $\left(f_{I K E, i,}, h_{I K E, i}\right)$ is used to derive fractional constituents of the master secret key $\left(M S K_{i, 1}, M S K_{i, 2}\right)$ involved in the $i$-th execution of Identity key extract algorithm. $\left(f_{T K E, j}, h_{T K E, j}\right)$ is used to derive fractional constituents of the cloud secret key $\left(C S K_{j, 1}, C S K_{j, 2}\right)$ involved in the $j$-th execution of Time update key extract algorithm. Furthermore, $\left(f_{\text {SIG }, k}\right.$, $\left.h_{S I G, k}\right)$ is applied to derive fractional constituents of the identity key $\left(I K_{I D, k, 1}, I K_{I D, k, 2}\right)$ and secret key $\left(S K_{I D, k, 1}\right.$, $\left.S K_{I D, k, 2}\right)$ involved in the $k$-th execution of Signing algorithm of a user ID. The private (or secret) keys leaked by the adversary in the associated computational algorithms are bounded to $\lambda$ bits, where $\lambda$ is a leakage parameter. Namely, the output lengths of $f_{\text {IKE, },}, h_{I K E, i}$, $f_{T K E, j,}, h_{T K E, j,}, f_{S I G, k}$ and $h_{S I G, k}$ are bounded to $\lambda$ bits, namely, $\left|f_{I K E, i}\right|,\left|h_{I K E, i}\right|,\left|f_{T K E, j}\right|,\left|h_{T K E, j}\right|,\left|f_{\text {SIG, }, k}\right|,\left|h_{S T G, k}\right| \leq \lambda$, where $\mid$ fun $\mid$ represents the output length of the function fun. The inputs/outputs of six leakage functions are presented as below.

- $\Lambda f_{\text {IKE,i }}=f_{I K E, i}\left(M S K_{i, 1}, R f_{I K E, i}\right)$.

- $\Lambda h_{I K E, i}=h_{I K E, i}\left(M S K_{i, 2}, R h_{I K E, i}\right)$.

- $\Lambda f_{T K E, j}=f_{T K E, j}\left(C S K_{j, 1}, R f_{T K E, j}\right)$.

- $\Lambda h_{T K E, j}=h_{T K E, j}\left(C S K_{j, 2}, R h_{T K E, j}\right)$.

- $A f_{\text {SIG,k }}=f_{S I G, k}\left(I K_{I D, k, k}, S K_{I D, k, 1}, R f_{S I G, k}\right)$.

- $\Lambda h_{S I G, k}=h_{S I G, k}\left(I K_{I D, k, 2}, S K_{I D, k, 2}, R h_{S I G, k)}\right)$.

Here, $R f_{I K E, i}, R h_{I K E, i}, R f_{T K E, j}, R h_{T K E, j,} R f_{S I G, k}$ and $R h_{S I G, k}$ represent the random values involved in the associated computational algorithms.

By extending the security notions in the RCLS and ORCLS schemes [7, 12, 21, 24], the adversary model of LR-ORCLS schemes contains three types of adversaries namely, Type I ( $A_{I}$, outsider), Type II $\left(A_{I I}\right.$, revoked user) and Type III ( $A_{I I}$, honest-but-curious $\mathrm{KGC})$. By providing the associated leak queries, three 
types of adversaries in the LR-ORCLS scheme are presented below.

- Type I adversary $\left(A_{I}\right.$, outsider): $A_{I}$ is permitted to retrieve the secret key $S K_{I D}$ and time update key $T K_{I D, t}$ of any user ID for any period $T_{t}$. However, $A_{I}$ does not know the master secret key $M S K$, but it is permitted to retrieve the identity key $I K_{I D}$ of any user $I D$, except the attacking target user $I D$. Additionally, $A_{I}$ can derive fractional constituent of the master secret key $M S K$ in Identity key extract algorithm. $A_{I}$ can also derive fractional constituent of $I K_{I D}$ in the Signing algorithm.

- Type II adversary ( $A_{I I}$, revoked user): $A_{I I}$ is permitted to retrieve the secret key $S K_{I D}$ and identity key $I K_{I D}$ of any user $I D$. However, $A_{I I}$ does not know the cloud secret key CSK, but it is permitted to retrieve the time update key $T K_{I D, t}$ of any user $I D$ for any period $T_{t}$, except $T K_{I D^{*} t^{*}}$ of the target user $I D^{*}$ at period $T_{t^{*}}$ Additionally, $A_{I I}$ can derive fractional constituent of CSK in the Time update key extract algorithm.

- Type III adversary $\left(A_{I I I}\right.$, honest-but-curious $\left.K G C\right)$ : $A_{I I I}$ is permitted to retrieve the identity key $I K_{I D}$ and time update key $T K_{I D, t}$ of any user ID for any period $T_{t}$. Additionally, it is permitted to retrieve the secret key $S K_{I D}$ of any user $I D$, except $S K_{I D^{*}}$ of the attacking target user $I D^{*}$. Meanwhile, $A_{I I I}$ can derive fractional constituent of the secret key $S K_{I D^{*}}$ in the Signing algorithm.

In the continual leakage model, the security notions of LR-ORCLS schemes are defined in the following security game played by both a challenger $C$ and an adversary $A\left(A_{I}, A_{I I}\right.$ or $\left.A_{I I I}\right)$.

Definition 2. In the continual leakage model, a LR-ORCLS scheme is existential unforgeable against adaptive chosen-message attacks (UF-LR-ORCLSACMA) if no adversary $A\left(A_{I}, A_{I I}\right.$ or $\left.A_{I I I}\right)$ wins the UFLR-ORCLS-ACMA game with non-negligible probability in polynomial time. This game includes three phases below:

- Setup: A challenger $C$ performs the Setup algorithm to produce the master secret key $M S K=\left(M S K_{0,1}\right.$, $\left.M S K_{0,2}\right)$ and cloud secret key $C S K=\left(C S K_{0,1}\right.$, $C S K_{0,2}$ ), and sets $z+1$ periods $T_{0}, T_{1}, \ldots, T_{z}$ and public parameters $P P$. According to adversary type, $C$ runs the following steps:

- If $A$ is of $A_{I}, C$ sends $P P$ and $C S K$ to $A$.

- If $A$ is of $A_{I I}, C$ sends $P P$ and $M S K$ to $A$.
- If $A$ is of $A_{I I I}, C$ sends $P P, M S K$ and $C S K$ to $A$.

- Queries: In this phase, $A$ can adaptively issue the following queries to $C$.

- Identity key query (ID): For the $i$-th execution, $C$ sets the new master secret key $\left(M S K_{i, 1}, M S K_{i, 2}\right)$ by using $\left(M S K_{i-1,1}, M S K_{i-1,2}\right)$. Afterward, $C$ uses $\left(M S K_{i, 1}, M S K_{i, 2}\right)$ to generate and return the associated identity key $I K_{I D}$.

- Identity key leak query $\left(f_{I K E, i}, h_{I K E, i}, i\right)$ : For the $i$-th Identity key query, $A$ is permitted to issue this query only once. $C$ returns fractional constituents $\left(\Lambda f_{\text {IKE, },}, \Lambda h_{\text {IKE, },}\right)$.

- Time update key query (ID, $T_{t}$ ): For the $j$-th execution, $C$ sets the current cloud secret key $\left(C S K_{j, 1}, C S K_{j, 2}\right)$ by using $\left(C S K_{j-1,1}, C S K_{j-1,2}\right)$. Afterward, $C$ uses $\left(C S K_{j, 1}, C S K_{j, 2}\right), I D$ and $T_{t}$ to generate and return the associated time update key $T K_{I D, t^{*}}$

- Time update key leak query $\left(f_{T K E, j,}, h_{T K E, j,}, j\right)$ : For the $j$-th Time update key query, $A$ is permitted to issue this query only once. $C$ returns fractional constituents $\left(\Lambda f_{T K E, j,}, \Lambda h_{T K E, j}\right)$.

- Public key retrieve query (ID, $T_{t}$ ): $C$ returns the associated public key tuple $\left(Q_{I D}, R_{I D, t}, P K_{I D}\right)$.

- Public key replace query $\left(I D, T_{t},\left(Q_{I D}^{\prime}, R_{I D, t}^{\prime}, P K_{I D}^{\prime}\right)\right)$ : $C$ records this public key replacement.

- Secret key corrupt query (ID): C returns the secret key $S K_{I D}$ if Public key replace query (ID) is never issued. Otherwise, $C$ returns false.

- Signing query (ID, $T_{t}, m s g$ ): For the $k$-th execution of $I D$ at period $T_{t}, C$ sets the current identity key $\left(I K_{I D, k, 1}, I K_{I D, k, 2}\right)$ and secret key $\left(S K_{I D, k, 1}, S K_{I D, k, 2}\right)$ by using $\left(I K_{I D, k-1,1,}, I K_{I D, k-1,2}\right)$ and $\left(S K_{I D, k-1,1,}, S K_{I D, k-1,2}\right)$, respectively. Afterward, $C$ uses $\left(I K_{I D, k, 1}, I K_{I D, k, 2}\right)$, $T K_{I D, t}$ and $\left(S K_{I D, k, 1}, S K_{I D, k, 2}\right)$ to generate and return a signature $\sigma$.

- Signing leak query (ID, $T_{t}, f_{\text {SIG }, k}, h_{S I G, k}, k$ ): For the $k$-th Signing query of $I D$ at period $T_{t}, A$ is permitted to issue this query only once. $C$ returns fractional constituents $\left(\Lambda f_{\text {SIG, },}, \Lambda h_{\text {SIG, }, k}\right)$.

- Forgery: In this phase, $A$ outputs a tuple (ID, $T_{t}^{*}$, $\left.m s g^{*}, \sigma^{*}\right)$. If the following conditions hold, it is said that $A$ wins the game.

1 Sign query $\left(I D^{*}, T_{t}^{*}, m s g^{*}\right)$ is never issued.

2 The response of the Verify algorithm on $\left(I D^{*}, T_{t}^{*}\right.$, $\left.m s g^{*}, \sigma^{*}\right)$ is "accept". 
3 According to the adversary type, $C$ checks the following conditions:

a If $A$ is of $A_{I}$, the Identity key query (ID") is never requested.

b If $A$ is of $A_{I I}$, the Time update key query (ID*, $T_{t}^{*}$ ) is never requested.

c If $A$ is of $A_{I I I}$, the Public key replace query (ID", $T_{t}^{*}$ ) or Secret key corrupt query (ID") is never requested.

\section{The Proposed LR-ORCLS Scheme}

The proposed LR-ORCLS scheme includes eight algorithms as below.

- Setup: The $K G C$ chooses the related parameters $\left\{G, G_{T}, p, P, \hat{e}\right\}$ of bilinear pairing groups presented in Section II.A. The $K G C$ then performs the following steps:

1 Choose a random integer $x \in Z_{p}^{*}$, and compute the master secret key $M S K=x \cdot P$ and master public key $M P K=\hat{e}(P, M S K)$.

2 Choose a random integer $y \in Z_{p}^{*}$, and compute the cloud secret key $C S K=y \cdot P$ and cloud public key $C P K=\hat{e}(P, C S K)$.

3 Choose a random integer $a_{0} \in Z_{p}^{*}$ and compute the primary master secret key $\left(M S K_{0,1}, M S K_{0,2}\right)$ $=\left(a_{0} \cdot P, M S K+\left(a_{0}\right) \cdot P\right)$.

4 Choose six random integers $r, s, u, v, m, n \in Z_{p}^{*}$, and compute $R=r \cdot P, S=s \cdot P, U=u \cdot P, V=v \cdot P, M=m \cdot P$, $N=n \cdot P$.

5 Choose $z+1$ periods $T_{t} \in\{0,1\}^{*}$, for $t=0,1, \ldots, z$.

6 Publish public parameters $P P=\{G, G, p, P, \hat{e}$, $M P K, C P K, R, S, U, V, M, N\}$.

7 Securely send CSK to the CRS. The CRS then chooses a random integer $b_{0} \in Z_{p}^{*}$ and sets the primary cloud secret key $\left(C S K_{0,1}, C S K_{0,2}\right)=\left(b_{0} \cdot P\right.$, $\left.C S K+\left(-b_{0}\right) \cdot P\right)$.

- Identity key extract: For the $i$-th execution, by taking as input a user $I D$, the $K G C$ runs two subalgorithms as below:

- IKExtract-1 $\left(M S K_{i-1,1}\right)$ :

1 Choose a random integer $a_{i} \in Z_{p}^{*}$, and compute $M S K_{i, 1}=M S K_{i-1,1}+a_{i} \cdot P$.

2 Choose a random integer $\alpha \in Z_{p}^{*}$, and compute $Q_{I D}=\alpha \cdot P$ and temporary information $T I_{I K E}=$ $M S K_{i, 1}+\alpha \cdot(R+I D \cdot S)$.
- IKExtract-1 ( $\left.\mathrm{MSK}_{\mathrm{i}-1,2}\right)$ :

1 Compute $M S K_{i, 2}=M S K_{i-1,2}+\left(-a_{i}\right) \cdot P \quad$ and $I K_{I D}=M S K_{i, 2}+T I_{I K E}$.

2 Send the user's identity key $I K_{I D}$ and partial public key $Q_{I D}$ to the user via a secure channel.

- Time update key extract: For the $j$-th execution, by taking a non-revoked user ID and the current period $T_{t}$ as input, the CRS runs two sub-algorithms as below:

- TKExtract-1 $\left(C S K_{j-1,1}\right)$ :

1 Choose a random integer $b_{j} \in Z_{p}^{*}$, and compute $C S K_{j, 1}=C S K_{j-1,1}+b_{j} P$.

2 Choose a random integer $\beta \in Z_{p}^{*}$, and compute $R_{I D, t}=\beta \cdot P$ and temporary information $T I_{T K E}=$ $C S K_{j, 1}+\beta \cdot\left(U+\left(I D \| T_{t}\right) \cdot V\right)$.

- TKExtract-1 $\left(C S K_{j-1,2}\right)$ :

1 Compute $C S K_{j, 2}=C S K_{j-1,2}+\left(-b_{j}\right) \cdot P$ and $T K_{I D, t}=$ $C S K_{j, 2}+T I_{T K E}$.

2 Send the user's time update key $T K_{I D, t}$ and partial public key $R_{I D, t}$ to the user via a public channel.

- Set secret key: A user ID randomly chooses an integer $z \in Z_{p}^{*}$ and computes her/his secret key $S K_{I D}=z \cdot P$ and partial public key $P K_{I D}=\hat{e}\left(P, S K_{I D}\right)$.

- Set private key: At period $T_{t}$, the signing private key of a user $I D$ consists of the identity key $I K_{I D}$, the time update key $T K_{I D, t}$ and the secret key $S K_{I D}$. The user runs the following steps:

1 Choose a random integer $c_{0} \in Z_{p}^{*}$ and compute the primary identity key $\left(I K_{I D, 0,1}, I K_{I D, 0,2}\right)=\left(c_{0} \cdot P, I K_{I D}\right.$ $\left.+\left(c_{0}\right) \cdot P\right)$.

2 Choose a random integer $d_{0} \in Z_{p}^{*}$ and compute the primary secret key $\left(S K_{I D, 0,1}, S K_{I D, 0,2}\right)=\left(d_{0} \cdot P, S K_{I D}\right.$ $\left.+\left(d_{0}\right) \cdot P\right)$.

3 Sets her/his primary private key tuple $\left(\left(I K_{I D, 0,1}\right.\right.$, $\left.\left.I K_{I D, 0,2}\right), T K_{I D, t,}\left(S K_{I D, 0,1}, S K_{I D, 0,2}\right)\right)$.

- Set public key: Upon receiving the partial public keys $Q_{I D}, R_{I D, t}$ and $P K_{I D}$, the user sets her/his public key tuple $\left(Q_{I D}, R_{I D, t}, P K_{I D}\right)$ at period $T_{t}$.

- Signing: For the $k$-th execution of a user ID at period $T_{t}$, by taking a message $m s g$ as input, the user runs two sub-algorithms as below:

- Signing-1 (IK $\left.{ }_{I D, k-1,1}, T K_{I D, t}, S K_{I D, k-1,1}\right)$ :

1 Randomly choose an integer $c_{k} \in Z_{p}^{*}$, and compute $I K_{I D, k, 1}=I K_{I D, k-1,1}+c_{k} \cdot P$. 
2 Randomly choose an integer $d_{k} \in Z_{p}^{*}$, and compute $S K_{I D, k, 1}=S K_{I D, k-1,1}+d_{k} \cdot P$.

3 Randomly choose an integer $\gamma \in Z_{p}^{*}$, and compute $\sigma_{1}=\gamma \cdot P$ and temporary information $T I_{S I G}=I K_{I D, k, 1}+T K_{I D, t}+S K_{I D, k, 1}+\gamma \cdot\left(M+\left(I D\left\|T_{t}\right\|\right.\right.$ $m s g) \cdot N)$.

- Signing-2 $\left(I K_{I D, k-1,2}, S K_{I D, k-1,2}\right)$ :

1 Compute $I K_{I D, k, 2}=I K_{I D, k-1,2}+\left(-c_{k}\right) \cdot P$ and $S K_{I D, k, 2}=$ $S K_{I D, k-1,2}+\left(-d_{k}\right) \cdot P$.

2 Compute $\sigma_{2}=I K_{I D, k, 2}+S K_{I D, k, 2}+T I_{S I G}$.

3 Set a signature tuple (ID, $T_{t}$, $\left.m s g, \sigma=\left(\sigma_{1}, \sigma_{2}\right)\right)$.

- Verifying: Given a signature tuple (ID, $T_{t}, m s g, \sigma=$ $\left.\left(\sigma_{1}, \sigma_{2}\right)\right)$ and the associated public key tuple $\left(Q_{I D}, R_{I D, t}\right.$, $\left.P K_{I D}\right)$, a verifier accepts it if $\hat{e}\left(P, \sigma_{2}\right)=M P K \cdot \hat{e}\left(Q_{I D}, R\right.$ $+I D \cdot S) \cdot C P K \cdot \hat{e}\left(R_{I D, t} U+\left(I D \| T_{t}\right) \cdot V\right) \cdot P K_{I D} \hat{e}\left(\sigma_{1}, M+\right.$ $\left.\left(I D\left\|T_{t}\right\| m s g\right) \cdot N\right)$. Otherwise, the verifier rejects it.

In the following, the signature correctness is demonstrated. By the key blinding (refreshing) technique, we have

$$
\begin{aligned}
& M S K=M S K_{0,1}+M S K_{0,2}=M S K_{1,1}+M S K_{1,2}=\ldots \\
& =M S K_{i, 1}+M S K_{i, 2} ; \\
& C S K=C S K_{0,1}+C S K_{0,2}=C S K_{1,1}+C S K_{1,2}=\ldots \\
& =C S K_{j, 1}+C S K_{j, 2} ; \\
& I K_{I D}=I K_{I D, 0,1}+I K_{I D, 0,2}=I K_{I D, 1,1}+I K_{I D, 1,2}=\ldots \\
& =I K_{I D, k, 1} I K_{I D, k, 2} ; \\
& S K_{I D}=S K_{I D, 0,1}+S K_{I D, 0,2}=S K_{I D, 1,1}+S K_{I D, 1,2}=\ldots \\
& =S K_{I D, k, 1}+S K_{I D, k, 2}
\end{aligned}
$$

Therefore, the signature correctness is demonstrated below.

$$
\begin{aligned}
& \hat{e}\left(P, \sigma_{2}\right)= \\
& \hat{e}\left(P, I K_{I D}+T K_{I D, t}+S K_{I D}+\gamma \cdot\left(M+\left(I D\left\|T_{t}\right\| m s g\right) \cdot N\right)\right) \\
& =\hat{e}\left(P, M S K+\alpha \cdot(R+I D \cdot S)+C S K+\beta \cdot\left(U+\left(I D \| T_{t}\right) \cdot V\right)\right. \\
& \left.+S K_{I D}+\gamma \cdot\left(M+\left(I D\left\|T_{t}\right\| m s g\right) \cdot N\right)\right) \\
& =\hat{e}(P, M S K) \cdot \hat{e}(P, \alpha \cdot(R+I D \cdot S)) \cdot \hat{e}(P, C S K) \cdot \hat{e}(P, \beta \cdot(U+ \\
& \left.\left.\left(I D \| T_{t}\right) \cdot V\right)\right) \cdot \hat{e}\left(P, S K_{I D}\right) \cdot \hat{e}\left(P, \gamma \cdot\left(M+\left(I D\left\|T_{t}\right\| m s g\right) \cdot N\right)\right) \\
& =M P K \cdot \hat{e}(\alpha \cdot P, R+I D \cdot S) \cdot C P K \cdot \hat{e}\left(\beta \cdot P, U+\left(I D \| T_{t}\right) \cdot V\right) \\
& \cdot P K_{I D} \cdot \hat{e}\left(\gamma \cdot P, M+\left(I D\left\|T_{t}\right\| m s g\right) \cdot N\right) \\
& =M P K \cdot \hat{e}\left(Q_{I D}, R+I D \cdot S\right) \cdot C P K \cdot \hat{e}\left(R_{I D, t}, U+\left(I D \| T_{t}\right) \cdot V\right) . \\
& P K_{I D} \cdot \hat{e}\left(\sigma_{1}, M+\left(I D\left\|T_{t}\right\| m s g\right) \cdot N\right) .
\end{aligned}
$$

\section{Security Analysis}

In this section, the security analysis of the proposed LR-ORCLS scheme is given. As the UF-LR-ORCLSACMA game presented in Definition 2, the adversary model includes three types of adversaries, namely, Type I ( $A_{I}$, outsider), Type II ( $A_{I I}$, revoked user) and Type III ( $A_{I I I}$, honest-but-curious KGC). In the GBG model, three theorems are, respectively, proved to demonstrate that our scheme is existential unforgeable against all Types I, II and III adversaries in the continual leakage model.

Theorem 1. In the GBG model, our LR-ORCLS scheme is existential unforgeable against Type I adversary $\left(A_{I}\right.$, outsider) in the UF-LR-ORCLS-ACMA game.

Proof. Let $A_{I}$ be of Type I adversary in the UF-LRORCLS-ACMA game played with a challenger $C . A_{I}$ may issue various queries to $C$ at most $q$ times in the game. In the GBG model, for performing three group operations, an adversary issues three associated queries $Q_{G}, Q_{T}$ and $Q_{p}$. In the game, there are three phases below:

- Setup phase: C first runs the Setup algorithm of the proposed LR-ORCLS scheme to generate $M S K$, $C S K, z+1$ periods $T_{0}, T_{1}, \ldots, T_{z}$ and $P P=\left\{G, G_{T}, p, P\right.$, $\hat{e}, M P K, C P K, R, S, U, V, M, N\}$. In the following, five lists $L_{G}, L_{G T}, L_{I K E}, L_{U}$ and $L_{T K E}$ are constructed to record both the inputs and outputs of queries issued by $A_{T}$.

- $\mathrm{L}_{\mathrm{G}}$ and $\mathrm{L}_{\mathrm{GT}}$ are, respectively, applied to record elements of two groups $\mathrm{G}$ and $\mathrm{G}_{\mathrm{T}}$.

$1 L_{G}$ includes pairs of $\left(\Xi G_{t, v, r}, \Theta G_{t, v, r}\right) . \Xi G_{t, v, r}$ is a multivariate polynomial to represent an element in $G$ and $\Theta G_{t, v, r}$ is the corresponding bit-string, where $t, v$ and $r$, respectively, represent the query type $t$, the $v$-th query and $r$-th element in $G$. Initially, $C$ stores nine pairs $\left(\Xi P, \Theta G_{I, 0,1}\right),\left(\Xi M S K, \Theta G_{I, 0,2}\right),\left(\Xi C S K, \Theta G_{I, 0,3}\right)$, $\left(\Xi R, \Theta G_{I, 0,4}\right),\left(\Xi S, \Theta G_{I, 0,5}\right),\left(\Xi U, \Theta G_{I, 0,6}\right),(\Xi V$, $\left.\Theta G_{I, 0,7,}\right),\left(\Xi M, \Theta G_{I, 0,8}\right)$ and $\left(\Xi N, \Theta G_{I, 0,9}\right)$ in $L_{G}$.

$2 L_{G T}$ includes pairs of $\left(\Xi T_{t, v, r}, \Theta T_{t, v, r}\right) . \Xi T_{t, v, r}$ is a multivariate polynomial to represent an element in $G_{T}$ and $\Theta G_{t, v, r}$ is the corresponding bitstring, where $t, v$ and $r$ have the same meanings in $L_{G}$. Initially, $C$ stores two pairs ( $\Xi M P K$, $\left.\Theta T_{I, 0,1}\right)$ and $\left(\Xi C P K, \Theta T_{I, 0,2}\right)$ in $L_{G T}$, where $\Xi M P K=\Xi P \cdot \Xi M S K$ and $\Xi C P K=\Xi P \cdot \Xi C S K$. 
Note that two transformation rules for $L_{G} / L_{G T}$ are given below.

1 On receiving a polynomial $\Xi G_{t, v, r}: \Xi T_{t, v, r}, C$ looks for $\left(\Xi G_{t, v, r}, \Theta G_{t, v, r}\right) /\left(\Xi T_{t, v, r}, \Theta T_{t, v, r}\right)$ in $L_{G} / L_{G T}$. If so, $C$ returns the bit-string $\Theta G_{t, v, r} / \Theta T_{t, v, r}$. Otherwise, $C$ randomly selects and returns a distinct bit-string $\Theta G_{t, v, r} / \Theta T_{t, v, r}$. Additionally, $C$ adds $\left(\Xi G_{t, v, r}, \Theta G_{t, v, r}\right) /$ $\left(\Xi T_{t, v, r}, \Theta T_{t, v, r}\right)$ in $L_{G} / L_{G T}$.

2 On receiving an encoded bit-string $\Theta G_{t, v, r} / \Theta T_{t, v, r}, C$ looks for $\left(\Xi G_{t, v, r}, \Theta G_{t, v, r}\right) /\left(\Xi T_{t, v, r}, \Theta T_{t, v, r}\right)$ in $L_{G} / L_{G T}$. If it is found, $C$ returns the associated multivariate polynomial $\Xi G_{t, v, r} / \Xi T_{t, v, r}$. Otherwise, $C$ terminates the game.

- $L_{I K E}$ includes tuples of (ID, $\Xi I K_{I D}, \Xi Q_{I D}$ ), where $\Xi I K_{I D}$ and $\Xi Q_{I D}$, respectively, denote the user's $I K_{I D}$ and $Q_{I D}$ in the Identity key extract algorithm.

- $L_{U}$ includes tuples of (ID, $\Xi S K_{I D}, \Xi P K_{I D}$ ), where $\Xi S K_{I D}$ and $\Xi P K_{I D}$, respectively, denote the user's $S K_{I D}$ and $P K_{I D}$ in the Set secret key algorithm.

- $L_{T K E}$ includes tuples of (ID, $T_{t}, \Xi T K_{I D, t}, \Xi R_{I D, t}$ ), where $\Xi T K_{I D, t}$ and $\Xi R_{I D, t}$, respectively, denote the user's $T K_{I D, t}$ and $R_{I D, t}$ in the Time update key extract algorithm.

Finally, $C$ sends these public parameters $\Xi P, \Xi R, \Xi S$, $\Xi U, \Xi V, \Xi M, \Xi N, \Xi M P K$ and $\Xi C P K$ to $A_{T}$. Meanwhile, $C$ sends the cloud secret key $\Xi C S K$ to $A_{I}$.

- Query phase: $A_{I}$ can adaptively request various queries to $C$ at most $q$ times. Note that since $A_{I}$ is permitted to get the secret key $S K_{I D}$ and time update key $T K_{I D, t}$ of any user ID for any period $T_{t}, A_{I}$ has no need to request the Public key replace query and Time update key leak query.

- $Q_{G}$ query $\left(\Theta G_{Q, l, 1}, \Theta G_{Q, l, 2}\right.$, Operation): For the $l$-th $Q_{G}$ query, $C$ runs the following steps.

1 Transform a pair of bit-strings $\left(\Theta G_{Q, l, 1}\right.$, $\left.\Theta G_{Q, l, 2}\right)$ to get a pair of polynomials $\left(\Xi G_{Q, l, 1}\right.$, $\left.\Xi G_{Q, l, 2}\right)$ in $L_{G^{*}}$.

2 Compute the resulting polynomial $\Xi G_{Q, l, 3}=$ $\Xi G_{Q, l, 1}+\Xi G_{Q, l, 2}$ if Operation= "addition", and $\Xi G_{Q, l, 3}=\Xi G_{Q, l, 1}-\Xi G_{Q, l, 2}$ if Operation="subtraction".

3 Transform $\Xi G_{Q, l, 3}$ to return the encoded bitstring $\Theta G_{Q, l, 3}$.

- $Q_{T}$ query $\left(\Theta T_{Q, l, 1}, \Theta T_{Q, l, 2}\right.$, Operation): For the $l$-th $Q_{T}$ query, $C$ runs the following steps.
1 Transform a pair of bit-strings $\left(\Theta T_{Q l, 1}\right.$, $\left.\Theta T_{Q, l, 2}\right)$ to get a pair of polynomials $\left(\Xi T_{Q, l, 1}\right.$, $\left.\Xi T_{Q, l, 2}\right)$ in $L_{G T}$.

2 Compute the resulting polynomial $\Xi T_{Q, l, 3}=$ $\Xi T_{Q, l, 1}+\Xi T_{Q, l, 2}$ if Operation="multiplication", and $\Xi T_{Q, l, 3}=\Xi T_{Q, l, 1}-\Xi T_{Q, l, 2}$ if Operation="division".

3 Transform $\Xi T_{Q, l, 3}$ to return the encoded bitstring $\Theta T_{Q, l, 3}$.

- $Q_{P}$ query $\left(\Theta G_{P, l, 1}, \Theta G_{P, l, 2}\right)$ : For the $l$-th $Q_{P}$ query, $C$ runs the following steps.

1 Transform a pair of bit-strings $\left(\Theta G_{P, l, 1}, \Theta G_{P,, 2}\right)$ to get a pair of polynomials $\left(\Xi G_{P, l, 1}, \Xi G_{P, l, 2}\right)$.

2 Compute the resulting polynomial $\Xi T_{P, l, 1}=$ $\mid \Xi G_{P, l, 1} \cdot \Xi G_{P, l, 2}$.

3 Transform $\Xi T_{P, l, 1}$ to return the encoded bitstring $\Theta T_{P, l, 1}$.

- Identity key query (ID): For the $i$-th execution, $C$ searches (ID, $\left.\Xi I K_{I D}, \Xi Q_{I D}\right)$ in $L_{I K E}$. If it is found, $C$ transforms $\Xi I K_{I D}$ and $\Xi Q_{I D}$ to return two encoded bit-strings $\Theta I K_{I D}$ and $\Theta Q_{I D}$ to $A_{I}$. Otherwise, $C$ adds a record in $L_{I K E}$ as below.

1 Choose a new variate $\Xi T G_{I K, i, 1}$ in $G$.

2 Setapolynomial $\Xi Q_{I D}=\Xi T G_{I K, i, 1}$ and $\Xi T I D=I D$.

3 Compute the user's identity key $\Xi I K_{I D}=$ $\Xi M S K+\Xi T G_{I K, i, 1} \cdot(\Xi R+\Xi S \cdot \Xi T I D)$ while adding $\left(I D, \Xi I K_{I D}, \Xi Q_{I D}\right)$ in $L_{I K E}$.

4 Transform and return two encoded bitstrings $\Theta I K_{I D}$ and $\Theta Q_{I D}$ to $A_{I}$.

- Identity key leak query $\left(f_{I K E, i}, h_{I K E, i}, i\right)$ : For the $i$-th Identity key query, $A_{I}$ is permitted to issue this query to $C$ only once. $C$ returns two outputs $\Lambda f_{I K E, i}$ and $\Lambda h_{I K E, i}$ to $A_{I}$, where $\Lambda f_{I K E, i}=f_{I K E, i}\left(M S K_{i, 1}, a_{i}, \alpha\right)$ and $\Lambda h_{I K E, i}=h_{I K E, i}\left(M S K_{i, 2}, a_{i}, T I_{I K E}\right)$.

- Time update key query (ID, $T_{t}$ ): For the $j$-th execution, $C$ searches (ID, $T_{t}, \Xi T K_{I D, t}, \Xi R_{I D, t}$ ) in $L_{T K E}$. If it is found, $C$ transforms $\Xi T K_{I D, t}$ and $\Xi R_{I D, t}$ to return two bit-strings $\Theta T K_{I D, t}$ and $\Theta R_{I D, t}$ to $A_{I}$. Otherwise, $C$ adds a record in $L_{T K E}$ as below.

1 Choose a new variate $\Xi T G_{T K, I D, j, 1}$ in $G$.

2 Set a polynomial $\Xi R_{I D, t}=\Xi T G_{T K, I D, j, 1}$ and $\Xi T T D$ $=I D \| T_{t}$.

3 Set the user's time update key $\Xi T K_{I D, t}=\Xi C S K$ $+\Xi T G_{T K, I D, j, 1} \cdot(\Xi U+\Xi V \cdot \Xi T T D)$ while adding $\left(I D, \Xi S K_{I D}, \Xi P K_{I D}\right)$ in $L_{T K E}$. 
4 Transform and return two encoded bitstrings $\Theta T K_{I D, t}$ and $\Theta R_{I D, t}$ to $A_{T}$.

- Time update key leak query $\left(f_{T K E, j}, h_{T K E, j}, j\right)$ : For the $j$-th Time update key query, $A_{I}$ is permitted to issue this query to $C$ only once. $C$ returns two leakage outputs $\Lambda f_{T K E, j}$ and $\Lambda h_{T K E, j}$ to $A_{I I}$, where $\Lambda f_{T K E, j}=f_{T K E, j}\left(C S K_{j, 1}, b_{j}, \beta\right)$ and $\Lambda h_{T K E, j}=h_{T K E, j}\left(C S K_{j, 2}\right.$, $\left.b_{j}, T I_{T K E}\right)$.

- Public key retrieve query (ID, $T_{t}$ ): $C$ applies ID and $T_{t}$ to search $L_{K E}, L_{U}$ and $L_{T K E}$ and then obtains the corresponding public key tuple $\left(\Xi Q_{I D}, \Xi R_{I D, t}\right.$, $\left.\Xi P K_{I D}\right)$. $C$ then transforms and returns a tuple of bit-strings $\left(\Theta Q_{I D}, \Theta R_{I D, t}, \Theta P K_{I D}\right)$ to $A_{I}$.

- Public key replace query (ID, $T_{t},\left(\Theta Q_{I D}^{\prime}, \Theta R_{I D, t}^{\prime}\right.$, $\left.\Theta P K_{I D}^{\prime}\right)$ ): $C$ first transforms a tuple of bitstrings $\left(\Theta Q_{I D}^{\prime}, \Theta R_{I D, t,}^{\prime}, \Theta P K_{I D}^{\prime}\right)$ to obtain the corresponding tuple of polynomials $\left(\Xi Q_{I D}^{\prime}\right.$, $\left.\Xi R_{I D, t}^{\prime}, \Xi P K_{I D}^{\prime}\right) . C$ replaces the related tuples with $\left(I D,-, \Xi Q_{I D}^{\prime}\right)$ in $L_{K E}$, $\left(I D,-, \Xi P K_{I D}^{\prime}\right)$ in $L_{U}$ and (ID, $\left.T_{t},-, \Xi R_{I D, t}\right)$ in $L_{T K E}$.

- Secret key corrupt query (ID): If Public key replace query (ID) is never issued, $C$ uses ID to search (ID, $\left.\Xi S K_{I D}, \Xi P K_{I D}\right)$ in $L_{U}$. $C$ transforms the secret key $\Xi S K_{I D}$ to return the bit-string $\Theta S K_{I D}$. Otherwise, $C$ runs the following steps.

1 Choose a new variate $\Xi T G_{S K, I D, 1}$ in $G$.

2 Set two polynomials $\Xi S K_{I D}=\Xi T G_{S K, I D, 1}$ and $\Xi P K_{I D}=\Xi P \cdot \Xi S K_{I D}$, and store (ID, $\Xi S K_{I D}$, $\left.\Xi P K_{I D}\right)$ in $L_{U}$.

3 Transform $\Xi S K_{I D}$ and $\Xi P K_{I D}$ to obtain two encoded bit-strings $\Theta S K_{I D}$ and $\Theta P K_{I D}$.

4 Return the bit-string $\Theta S K_{I D}$ to $A_{I}$.

- Singing query (ID, $T_{t}, m s g$ ): For the $k$-th execution of the user $I D$ at period $T_{t}$, by taking as input $m s g$, $C$ runs the following steps.

1 By ID, search (ID, $\left.\Xi I K_{I D}, \Xi Q_{I D}\right)$ in $L_{I K E}$.

2 By $I D$, search (ID, $\left.\Xi S K_{I D}, \Xi P K_{I D}\right)$ in $L_{U}$.

3 By $I D$ and $T_{t}$, search (ID, $\left.T_{t}, \Xi T K_{I D, t}, \Xi R_{I D, t}\right)$ in $L_{T K E}$.

4 Choose a new variate $\Xi T G_{S, k, 1}$ in $G$ and set $\Xi \sigma_{1}=\Xi T G_{S, k, 1}$.

5 Set $\Xi \sigma_{2}=\Xi I K_{I D}+\Xi T K_{I D, t}+\Xi S K_{I D}+\Xi T G_{S, k, 1} \cdot(\Xi M$ $\left.+\left(I D\left\|T_{t}\right\| m s g\right) \cdot \Xi N\right)$.

6 Transform $\left(\Xi \sigma_{1}, \Xi \sigma_{2}\right)$ to gain and return the encoded bit-strings $\left(\Theta \sigma_{1}, \Theta \sigma_{2}\right)$ to $A_{I}$.
- Signing leak query (ID, $\left.T_{t}, f_{\text {SIG }, k}, h_{\text {SIG }, k}, k\right)$ : For the $k$-th Signing query of the user ID at period $T_{t}$, by taking as input two leakage functions $f_{\text {SIG, }}$ and $h_{S I G, k}, C$ returns $\Lambda f_{\text {SIGG, }}$ and $\Lambda h_{S I G, k}$ to $A_{I}$, where $\Lambda f_{S I G, k}=f_{S I G, k}\left(I K_{I D, k, 1}, S K_{I D, k, 1}, c_{k}, d_{k}, \gamma\right)$ and $\Lambda h_{S I G, k}=h_{S I G, k}$ $\left(I K_{I D, k, 2}, S K_{I D, k, 1}, c_{k}, d_{k}, T I_{S I G}\right)$. Note that $A_{I}$ is permitted to issue this query only once.

- Forgery phase: $A_{I}$ outputs $\left(I D^{*}, T_{t}^{*}, m s g^{*},\left(\Theta \sigma_{1}^{*}, \Theta \sigma_{2}^{*}\right)\right)$. $A_{I}$ is not permitted to issue the Signing query (ID", $T_{t}^{*}, m s g^{*}$ ) or Identity key query (ID"). C transforms $\left(\Theta \sigma_{1}^{*}, \Theta \sigma_{2}^{*}\right)$ to gain $\left(\Xi \sigma_{1}^{*}\right.$, and $\left.\Xi \sigma_{2}^{*}\right)$, and sets $T I D^{*}=I D^{*}$ and $T T D^{*}=I D^{*} \| T_{t}^{*}$. If the equality $\Xi P \cdot \Xi \sigma_{2}^{*}=\Xi M P K$ $+\Xi Q_{I D} \cdot(\Xi R+T I D * \Xi S)+\Xi C P K+\Xi R_{I D, t} \cdot(\Xi U+$ $\left.T T D^{*} \cdot \Xi V\right)+P K_{I D}+\Xi \sigma_{1}^{*} \cdot\left(\Xi M+\left(I D^{*}\left\|T_{t}^{*}\right\| m s g^{*}\right) \cdot \Xi N\right)$ holds, we say that $A_{I}$ wins the game.

In the following, let us evaluate the probability that $A_{I}$ wins the game. Firstly, the amounts of group elements in $L_{G}$ and $L_{G T}$ are counted as given below:

1 In the Setup phase, 9 and 2 elements are, respectively, added in $L_{G}$ and $L_{G T}$.

2 In the Query phase, the added amounts of $L_{G}$ and $L_{G T}$ for each query are discussed as follows.

- For each $Q_{G}, Q_{T}$ or $Q_{P}$ query, 3 elements could be added in $L_{G}$ or $L_{G T}$.

- For each Identity key query, 2 elements could be added in $L_{G}$.

- For each Time update key query, 2 elements could be added in $L_{G}$.

- For each Signing query, 8 elements could be added in $L_{G}$.

Let $q_{O}$ denote the total number of $Q_{G}, Q_{T}$ and $Q_{P}$ queries. Let $q_{I K}, q_{T K}$ and $q_{S}$, respectively, be the query numbers of the Identity key query, Time update key query and Signing query. Since $A_{I}$ is permitted to request all queries at most $q$ times, we have $\left|L_{G}\right|+$ $\left|L_{G T}\right| \leqq 11+3 q_{O}+2 q_{I K}+2 q_{T K}+8 q_{S} \leqq 8 q$.

Secondly, let us evaluate the maximal degrees of polynomials in $L_{G}$ and $L_{G T}$, respectively.

1 In $L_{G}$, the maximal degree of polynomials is 3 by the following discussions.

- In the Setup phase, nine new variates (polynomials) $\Xi P, \Xi M S K, \Xi C S K, \Xi R, \Xi S, \Xi U, \Xi V$, $\Xi M$ and $\Xi N$ are initially added in $L_{G}$. All these polynomials have degree 1 .

- For the $Q_{G} q u e r y, \Xi G_{Q, l, 3}$ has the maximal degree of 
$\Xi G_{Q, l, 1}$ or $\Xi G_{Q, l, 2}$.

- For the Identity key query, three polynomials $\Xi T G_{I K, i, 1}, \Xi T I D$ and $\Xi I K_{I D}$ have degrees 1,1 and 3 , respectively.

- For the Time update key query, three polynomials $\Xi T G_{T K, I D, j, 1}, \Xi T T D$ and $\Xi T K_{I D, t}$ have degrees 1, 1 and 3 , respectively.

- For the Signing query, two polynomials $\Xi \sigma_{1}$ and $\Xi \sigma_{2}$ have degrees 1 and 3 , respectively.

2 In $L_{G T}$, the maximal degree of polynomials is 6 by the following discussions.

- In the Setup phase, two polynomials $\Xi M P K$ and $\Xi C P K$ have degree 2 .

- For $Q_{T}$ query, $\Xi T_{Q, l, 3}$ has the maximal degree of $\Xi T_{Q, l, 1}$ or $\Xi T_{Q, l, 2}$.

- For $Q_{P}$ query, since the maximal degree of polynomials in $L_{G}$ is 3 and $\Xi T_{P, l, 1}=\Xi G_{P, l, 1} \cdot \Xi G_{P, l, 2}$, the polynomial $\Xi T_{P, l, 1}$ has degree 6 .

Let us evaluate the advantage that $A_{I}$ wins the game without requesting the Identity key leak query and Signing leak query. Subsequently, the advantage of $A_{I}$ with requesting two kinds of leak queries is evaluated.

1 The advantage of $A_{I}$ without requesting two kinds of leak queries: It is said that $A_{I}$ wins the game if anyone of two cases occurs.

Case 1: $A_{I}$ discovers a collision of any two elements in $L_{G}$ or $L_{G T}$. Firstly, let us evaluate the collision probability in $L_{G}$. Let $n$ denote the total number of all variates in $L_{G}$. The challenger $C$ selects $n$ random values $v_{l} \in Z_{p}^{*}$ for $l=1,2, \ldots, n$. Let $\Xi G_{i}$ and $\Xi G_{j}$ denote any two distinct polynomials in $L_{G}$. $C$ then computes $\Xi G_{C}=\Xi G_{i}-\Xi G_{j}$ and $\Xi G_{C}\left(v_{1}, v_{2}, \ldots, v_{n}\right)$. If $\Xi G_{C}\left(v_{1}, v_{2}, \ldots, v_{n}\right)=0$, it is said that the collision occurs. By Lemma 2 , the probability of $\Xi G_{C}\left(v_{1}, v_{2}, \ldots\right.$, $\left.v_{n}\right)=0$ is at most $3 / p$ because the maximal polynomial degree in $L_{G}$ is 3 and no fractional constituent $(\lambda=0)$ is leaked. Since there are $\left(\begin{array}{c}\left|L_{G}\right| \\ 2\end{array}\right)$ distinct pairs $\left(\Xi G_{i}, \Xi G_{j}\right)$ in $L_{G}$, the collision probability is $(3 / p)$ $\left(\begin{array}{c}\left|L_{G}\right| \\ 2\end{array}\right)$. For the collision probability in $L_{G T}$, by similar evaluations, it is $(6 / p)\left(\begin{array}{c}\left|L_{G T}\right| \\ 2\end{array}\right)$. As mentioned earlier, we have $\left|L_{G}\right|+\left|L_{G T}\right| \leqq 8 q$. Let the probability of Case 1 is denoted by $\operatorname{Pr}[$ Case 1], we have

$$
\begin{gathered}
\operatorname{Pr}[\text { Case } 1] \leqq(3 / p)\left(\begin{array}{c}
\left|L_{G}\right| \\
2
\end{array}\right)+(6 / p)\left(\begin{array}{c}
\left|L_{G T}\right| \\
2
\end{array}\right) \leqq \\
(6 / p)\left(\left|L_{G}\right|+\left|L_{G T}\right|\right)^{2} \leqq 384 q^{2} / p .
\end{gathered}
$$

Case 2: Let us evaluate the probability that $A_{I}$ outputs a valid signature (ID, $T_{t}^{*}, m s g^{*},\left(\Theta \sigma_{1}^{*}\right.$, $\left.\left.\Theta \sigma_{2}^{*}\right)\right)$ that satisfies $\Xi f=\Xi M P K+\Xi Q_{I D} \cdot\left(\Xi R+T I D^{*}\right.$. $\left.\Xi S)+\Xi C P K+\Xi R_{I D, t} \Xi U+T T D^{*} \cdot \Xi V\right)+P K_{I D}+$ $\Xi \sigma_{1}^{*} \cdot\left(\Xi M+\left(I D^{*}\left\|T_{t}^{*}\right\| m s g^{*}\right) \cdot \Xi N\right)-\Xi P \cdot \Xi \sigma_{2}^{*}=0$. Obviously, the degree of $\Xi f$ is at most 5 . By Lemma 2 , the probability is $5 / p$. Let the probability of Case 2 is denoted by $\operatorname{Pr}$ [Case 2], we have $\operatorname{Pr}[$ Case 2] $\leqq 5 / p$.

Let $A d v_{A I-W}$ is the advantage that $A_{I}$ wins the game without requesting two kinds of leak queries. By $\operatorname{Pr}$ [Case 1] and $\operatorname{Pr}$ [Case 2], we have

$$
\begin{gathered}
A d v_{A I-W} \leqq \operatorname{Pr}[\text { Case 1] }+\operatorname{Pr}[\text { Case } 2] \\
\leqq 384 q^{2} / p+5 / p=O\left(q^{2} / p\right) .
\end{gathered}
$$

Hence, $A d v_{A I-W}$ is negligible if $q=$ poly $(\log p)$.

\section{The advantage of $A_{I}$ with requesting two kinds} of leak queries: Firstly, let us discuss the fractional constituents of the private (or secret) keys involved in the associated leak queries.

1 Identity key leak query $\left(f_{I K E, i}, h_{I K E, i,}, i\right)$ : As mentioned earlier, we have the conditions $\left|f_{I K E, i}\right| \leq \lambda$ and $\left|h_{I K E, i}\right| \leq \lambda$. By this query, $A_{I}$ derives fractional constituents $\Lambda f_{I K E, i}=f_{I K E, i}\left(M S K_{i, 1}, a_{i}, \alpha\right)$ and $\Lambda h_{I K E, i}=h_{I K E, i}\left(M S K_{i, 2}, a_{i}, T I_{I K E}\right)$ that are discussed as below.

- $a_{i}, \alpha$ : Since $a_{i}$ and $\alpha$ are randomly selected in each Identity key query, the leakage information of $a_{i}$ or $\alpha$ is no help to learn the master secret key $M S K$.

- $\left(M S K_{i 1}, M S K_{i, 2}\right)$ : Indeed, the master secret key $M S K$ satisfies $M S K=M S K_{0,1}+M S K_{0,2}=$ $M S K_{1,1}+M S K_{1,2}=\ldots=M S K_{i, 1}+M S K_{i, 2}$. By the blinding technique, fractional constituent of $M S K_{i-1,1} / M S K_{i-1,2}$ is independent of that of $M S K_{i, 1} / M S K_{i, 2}$. Hence, $A_{I}$ derives at most $2 \lambda$ bits of $M S K$.

- $T I_{I K E}: T I_{I K E}$ is a temporary value and applied to compute the user's identity key $I K_{I D}$. Since $A_{I}$ can obtain the whole $I K_{I D}$ except for $I D^{*}, T I_{I K E}$ is helpless for $A_{I}$.

2 Signing leak query $\left(I D^{*}, T_{t}, f_{S I G, k}, h_{S I G, k}, k\right)$ : As mentioned earlier, we have the conditions $\left|f_{S I G, k}\right| \leq \lambda$ and $\left|h_{S I G, k}\right| \leq \lambda$. And $A_{I}$ is permitted to get the secret key $S K_{I D}$ and time update key $T K_{I D, t}$ of any user $I D$ for any period $T_{t}$. By this query, $A_{I}$ derives fractional constituents $\Lambda f_{\text {SIG, }}=$ 
$f_{S I G, k}\left(I K_{I D^{*}, k, 1}, c_{k}, d_{k}, \gamma\right)$ and $\Lambda h_{S I G, k}=h_{S I G, k}\left(I K_{I D^{*}, k, 2}, c_{k}\right.$, $\left.d_{k}, T I_{S I G}\right)$ that are discussed as below.

- $c_{k}, d_{k}, \gamma$ : Since $c_{k}, d_{k}$ and $\gamma$ are randomly selected in each signing query, their leakages are no help to learn the master secret key $I K_{I D^{*}}$

- $\left(I K_{I D^{*} k, 1,}, I K_{I D^{*}, k-1,2}\right)$ : Indeed, the identity key $I K_{I D^{*}}$ satisfies $I K_{I D^{*}}=I K_{I D^{*}, 0,1}+I K_{I D^{*}, 0,2}=I K_{I D^{*}, 1,1}+I K_{I D^{*}, 1,2}$ $=. . .=I K_{I D^{*}, k, 1}+I K_{I D^{*}, k, 2}$. By the blinding technique, fractional constituent of $I K_{I D^{*} k-1,1} / I K_{I D^{*} k-1,2}$ is independent of that of $I K_{I D^{*} k, 1} / I K_{I D^{*}, k, 2}$. Hence, $A_{I}$ derives at most $2 \lambda$ bits of $I K_{I D^{*}}$

- $T I_{\text {SIG }}: T I_{\text {SIG }}$ is a temporary value and applied to compute the signature $\sigma_{2}$. Since $A_{I}$ can obtain the entire $\sigma_{2}$ by the Sign query, $T I_{S I G}$ is helpless for $A_{I}$.

Let $A d v_{A I}$ be the advantage that $A_{I}$ wins the game with requesting the Identity key leak query and Signing leak query. If $A_{I}$ can know the master secret key $M S K$ or the target user's identity key $I K_{I D^{*}} A_{I}$ may forge a legal signature. Two events are defined as below.

1 Let the event EMSK denote that $A_{I}$ knows the whole $M S K$ by $\Lambda f_{I K E, i}$ and $\Lambda h_{I K E, i}$ while $\overline{E M S K}$ is the corresponding complement event.

2 Let the event $E I K$ denote that $A_{I}$ knows the whole $I K_{I D^{*}}$ by $\Lambda f_{\text {SIG }, k}$ and $\Lambda h_{\text {SIG }, k}$ while $\overline{E I K}$ is the corresponding complement event.

Let the event $E F S$ denote that $A_{I}$ can forge a legal signature. Hence, the advantage $A d v_{A I}$ is $\operatorname{Pr}[E F S]$ such that the following inequality

$$
\begin{aligned}
& A d v_{A I}=\operatorname{Pr}[E F S] \\
&=\operatorname{Pr}[E F S \wedge(E M S K \vee E I K)] \\
&+\operatorname{Pr}[E F S \wedge(\overline{E M S K} \wedge \overline{E I K})] \\
&=\operatorname{Pr}[E F S \wedge E M S K]+\operatorname{Pr}[E F S \wedge E I K] \\
&+\operatorname{Pr}[E F S \wedge \overline{E M S K} \wedge \overline{E I K}] \\
& \leqq \\
& \operatorname{Pr}[E M S K]+\operatorname{Pr}[E F S \wedge E I K] \\
& \leqq \operatorname{Pr}[E F S \wedge \overline{E M S K} \wedge \overline{E I K}] \\
&+\operatorname{Pr}[E F S \wedge \overline{E M S K} \wedge \overline{E I K}] .
\end{aligned}
$$

In Case 1 of $A_{I}$ without requesting two kinds of leak queries, the advantage is $\operatorname{Pr}$ [Case 1] $\leqq 384 q^{2} / p=$ $O\left(q^{2} / p\right)$. By the Identity key leak query, $A_{I}$ derives at most $2 \lambda$ bits of $M S K$. By Lemma 2 , we have $\operatorname{Pr}[E M S K] \leqq O\left(\left(q^{2} / p\right)^{*} 2^{2 \lambda}\right)$. By the similar reason, we have $\operatorname{Pr}[E I K] \leqq O\left(\left(q^{2} / p\right)^{*} 2^{2 \lambda}\right)$. Finally, the event $\overline{E M S K}$ $\wedge \overline{E I K}$ is that $A_{I}$ can get fractional constituents of $M S K$ and $I K_{I D^{*}}$. Since $A d v_{A I-W} \leqq O\left(q^{2} / p\right)$ and $A_{I}$ can gain at most $2 \lambda$ bits about $M S K$ and $I K_{I D}$, we have $\operatorname{Pr}[E F S \wedge$ $\overline{E M S K} \wedge \overline{E I K}] \leqq O\left(\left(q^{2} / p\right)^{*} 2^{2 \lambda}\right)$. According to the discussions above, we have

$$
\begin{aligned}
& A d v_{A I}=\operatorname{Pr}[E F S] \\
& \leqq \operatorname{Pr}[E M S K]+\operatorname{Pr}[E I K]+\operatorname{Pr}[E F S \wedge \overline{E M S K} \wedge \overline{E I K}] \\
& \leqq O\left(\left(q^{2} / p\right)^{*} 2^{2 \lambda}\right) .
\end{aligned}
$$

By Corollary 1, $A d v_{A I}$ is negligible if $\lambda<\log p-$ $\omega(\log \log p)$. Q.E.D.

Theorem 2. In the GBG model, our LR-ORCLS scheme possesses existential unforgeability against Type II adversary ( $A_{I I}$, revoked user) in the UF-LRORCLS-ACMA game.

Proof. Let $A_{I I}$ be of Type II adversary in the UF-LRORCLS-ACMA game played with a challenger $C . A_{I I}$ may issue various queries to $C$ at most $q$ times in the game. This game consists of three phases as follows:

- Setup Phase: The phase is the same with that of the proof in Theorem 1. $C$ sends the public parameters $\Xi P, \Xi R, \Xi S, \Xi U, \Xi V, \Xi M, \Xi N, \Xi M P K$ and $\Xi C P K$ to $A_{I}$. Additionally, $C$ also sends the master secret key $\Xi M S K$ to $A_{I I}$.

- Query phase: In this phase, $A_{I I}$ can adaptively issue various queries to $C$ at most $q$ times. All queries are identical to those queries in Theorem 1 . Note that since $A_{I I}$ is permitted to get both the identity key $I K_{I D}$ and secret key $U S K_{I D}$ of any user $I D, A_{I I}$ has no need to issue the Identity key leak query and Public key replace query. Indeed, a revoked user's time update key $U T K_{I D, t}$ is never generated so that the Signing leak query does not leak any content. Additionally, $A_{I I}$ can derive fractional constituents of the cloud secret key CSK by the Time update key leak query.

- Forgery phase: $A_{I I}$ outputs (ID, $T_{t}^{*}, \quad m s g^{*}$, $\left.\left(\Theta \sigma_{1}^{*}, \Theta \sigma_{2}^{*}\right)\right) . A_{I I}$ is not permitted to issue the Signing query (ID, $T_{t}^{*}, \mathrm{msg}^{*}$ ) or Time update key query $\left(I D^{*}, T_{t}^{*}\right) . C$ transforms $\left(\Theta \sigma_{1}^{*}, \Theta \sigma_{2}^{*}\right)$ to gain $\left(\Xi \sigma_{1}^{*}\right.$, and $\left.\Xi \sigma_{2}^{*}\right)$, and sets $T I D^{*}=I D^{*}$ and $T T D^{*}=I D^{*} \| T_{t}^{*}$. If the equality $\Xi P \cdot \Xi \sigma_{2}^{*}=\Xi M P K+$ $\Xi Q_{I D} \cdot(\Xi R+T I D * \Xi S)+\Xi C P K+\Xi R_{I D, t} \cdot(\Xi U$ $\left.+T T D^{*} \cdot \Xi V\right)+P K_{I D}+\Xi \sigma_{1}^{*} \cdot\left(\Xi M+\left(I D^{*}\left\|T_{t}^{*}\right\| m s g^{*}\right) \cdot \Xi N\right)$ holds, we say that $A_{I I}$ wins the game. 
In the following, let us evaluate the probability that $A_{I I}$ wins the game. Let us first evaluate the advantage that $A_{I I}$ wins the game without requesting the Time update key leak query. Subsequently, the advantage of $A_{I I}$ with requesting the Time update key leak query is evaluated.

1 The advantage of $A_{I I}$ without requesting the Time update key leak query: Let $A d v_{A I I-W}$ be the advantage that $A_{I I}$ wins the game without requesting the Time update key leak query. As the similar evaluations in Theorem 1, we have $A d v_{A I I-W}$ $=O\left(q^{2} / p\right)$.

2 The advantage of $A_{I I}$ with requesting the Time update key leak query: For the $j$-th Time update key leak query with $f_{T K E, j}$ and $h_{T K E, j}$ such that $\left|f_{T K E, j}\right| \leq \lambda$ and $\left|h_{T K E, j}\right| \leq \lambda, A_{I I}$ can gain fractional constituents $\Lambda f_{T K E, j}=f_{T K E, j}\left(C S K_{j, 1}, b_{j}, \beta\right)$ and $\Lambda h_{T K E, j}=h_{T K E, j}\left(C S K_{j, 2}\right.$, $\left.b_{j}, T I_{T K E}\right)$. Indeed, the cloud secret key CSK satisfies $C S K=C S K_{0,1}+C S K_{0,2}=C S K_{1,1}+C S K_{1,2}=\ldots=C S K_{j, 1}+$ $C S K_{j, 2}$. By the blinding technique, fractional constituent of $C S K_{j-1,1} / C S K_{j-1,2}$ is independent of that of $C S K_{j, 1} / C S K_{j, 2}$. In such a case, $A_{I I}$ derives at most $2 \lambda$ bits of CSK.

Let $A d v_{A I I}$ be the advantage that $A_{I I}$ wins the game with requesting the Time update key leak query. If $A_{I I}$ knows the whole cloud secret key $C S K, A_{I I}$ can get the time update key $T K_{I D^{*}, t^{*}}$ of the target user $I D^{*}$ at period $T_{t^{*}}$ Thus, $A_{I I}$ may forge a legal signature. Let the event $E C S K$ denote that $A_{I I}$ knows the whole $C S K$ by $f_{T K E, j}$ and $h_{T K E, j}$ while $\overline{E C S K}$ is the corresponding complement event. Let the event $E F S$ denote that $A_{I I}$ can forge a legal signature. Hence, we have $A d v_{A I I}=\operatorname{Pr}[E F S]$ that satisfies the following inequality

$$
\begin{aligned}
& A d v_{A I I}=\operatorname{Pr}[E F S] \\
& =\operatorname{Pr}[E F S \wedge E C S K]+\operatorname{Pr}[E F S \wedge \overline{E C S K}] \\
& \leqq \operatorname{Pr}[E C S K]+\operatorname{Pr}[E F S \wedge \overline{E C S K}] .
\end{aligned}
$$

By the Time update key leak query, $A_{I I}$ derives at most $2 \lambda$ bits of CSK. By Pr[Case 1] $\leqq O\left(\left(q^{2} / p\right)\right.$ in Theorem 1 and Lemma 2 , we have $\operatorname{Pr}[E C S K] \leqq O\left(\left(q^{2} / p\right)^{*} 2^{2 \lambda}\right)$. Finally, the event $\overline{E C S K}$ is that $A_{I I}$ can get fractional constituents of $\left(C S K_{j, 1}, C S K_{j, 2}\right)$ by $\Lambda f_{T K E, j}$ and $\Lambda h_{T K E, j}$. Since $A d v_{\text {AII-W }} \leqq O\left(q^{2} / p\right)$ and $A_{I I}$ can gain at most $2 \lambda$ bits about $C S K$, we have $\operatorname{Pr}[E F S \wedge \overline{E C S K}] \leqq O\left(\left(q^{2} / p\right)^{*} 2^{2 \lambda}\right)$. According to the discussions above, we have
$A d v_{A I I} \leqq \operatorname{Pr}[E C S K]+\operatorname{Pr}[E F S \wedge \overline{E C S K}] \leqq O\left(\left(q^{2} / p\right)^{*} 2^{2 \lambda}\right)$.

By Corollary 1, $A d v_{A I I}$ is negligible if $\lambda<\log p-\omega$ (loglog $p$ ). Q.E.D.

Theorem 3. In the GBG model, our LR-ORCLS scheme is existential unforgeable against Type III adversary $\left(A_{I I}\right.$, honest-but-curious $\left.K G C\right)$ in the UF-LRORCLS-ACMA game.

Proof. Let $A_{I I I}$ be of Type III adversary in the UFLR-ORCLS-ACMA game played with a challenger $C$. $A_{I I I}$ may issue various queries to the challenger $C$ at most $q$ times in the game. This game consists of three phases as follows:

- Setup Phase: The phase is the same with that of the proof in Theorem 1. $C$ sends public parameters $\Xi P, \Xi R, \Xi S, \Xi U, \Xi V, \Xi M, \Xi N, \Xi M P K$ and $\Xi C P K$ to $A_{I}$. Additionally, $C$ also sends the master secret key $\Xi M S K$ and the cloud secret key $\Xi C S K$ to $A_{I I I}$.

- Query phase: In this phase, $A_{I I I}$ can adaptively issue various queries to $C$ at most $q$ times. Note that since $A_{I I I}$ is permitted to get the identity key $I K_{I D}$ and time update key $T K_{I D, t}$ of any user $I D$ for any period $T_{t}, A_{I I I}$ has no need to issue the Identity key leak query and Time update key leak query. Additionally, $A_{I I I}$ is permitted to get the secret key $S K_{I D}$ of any user $I D$, except $S K_{I D^{*}}$ of the attacking target user ID*. Meanwhile, $A_{I I I}$ can derive fractional constituent of the secret key $S K_{I D^{*}}$ by the Signing leak query.

- Forgery phase: $A_{I I I}$ outputs (ID*, $T_{t}^{*}, m s g^{*},\left(\Theta \sigma_{1}^{*}\right.$, $\left.\left.\Theta \sigma_{2}^{*}\right)\right) . A_{I I I}$ is not permitted to issue the Signing query (ID*, $\left.T_{t}^{*}, m s g^{*}\right)$, Public key replace query (ID*, $T_{t}^{*}$ ) or Secret key corrupt query (ID"). C transforms $\left(\Theta \sigma_{1}^{*}, \Theta \sigma_{2}^{*}\right)$ to gain $\left(\Xi \sigma_{1}^{*}\right.$, and $\left.\Xi \sigma_{2}^{*}\right)$, and sets $T I D^{*}=I D^{*}$ and $T T D^{*}=I D^{*} \| T_{t}^{*}$. If the equality $\Xi P \cdot \Xi \sigma_{2}^{*}=\Xi M P K$ $+\Xi Q_{I D} \cdot\left(\Xi R+T I D^{*} \cdot \Xi S\right)+\Xi C P K+\Xi R_{I D, t} \cdot(\Xi U+$ $\left.T T D^{*} \cdot \Xi V\right)+P K_{I D}+\Xi \sigma_{1}^{*} \cdot\left(\Xi M+\left(I D^{*}\left\|T_{t}^{*}\right\| m s g\right) \cdot \Xi N\right)$ holds, it is said that $A_{I I I}$ wins the game.

In the following, let us evaluate the probability that $A_{I I I}$ wins the game. Let us evaluate the advantage that $A_{I I I}$ wins the game without requesting the Signing leak query. Subsequently, the advantage of $A_{I I I}$ with requesting the Signing leak query is evaluated.

1 The advantage of $A_{I I I}$ without requesting the Signing leak query: Let $A d v_{A I I I-W}$ is the advantage 
that $A_{I I}$ wins the game without requesting the Signing leak query. As the similar evaluations in Theorem 1, we have $A d v_{\text {AIII-W }}=O\left(q^{2} / p\right)$.

2 The advantage of $A_{I I I}$ with requesting the Signing leak query: For the $k$-th Signing leak query with $f_{\text {SIG }, k}$ and $h_{\text {SIG, }, k}$ such that $\left|f_{\text {SIGG, }}\right| \leq \lambda$ and $\left|h_{S I G, k}\right| \leq \lambda, A_{I I I}$ can get fractional constituents $\Lambda f_{S I G, k}=f_{S I G, k}\left(S K_{I D^{*}, k, 1}, c_{k}, d_{k}, \gamma\right)$ and $\Lambda h_{S I G, k}=h_{S I G, k}\left(S K_{I D^{*}, k, 1}\right.$, $\left.c_{k}, d_{k}, T I_{S I G}\right)$. Indeed, the user's secret key $S K_{I D^{*}}$ satisfies $S K_{I D^{*}}=S K_{I D^{*}, 0,1}+S K_{I D^{*}, 0,2}=S K_{I D^{*}, 1,1}+S K_{I D^{*}, 1,2}=\ldots$ $=S K_{I D^{*}, k, 1}+S K_{I D^{*}, k, 2}$. By the blinding technique, fractional constituent of $S K_{I D^{*}, k-1,1} / S K_{I D^{*} k-1,2,}$ is independent of that of $S K_{I D^{*} k, 1} / S K_{I D^{*}, k, 2}$. In such a case, $A_{I I I}$ derives at most $2 \lambda$ bits of $S K_{I D^{*}}$

Let $A d v_{\text {AIII }}$ be the advantage that $A_{I I I}$ wins the game with requesting the Signing leak query. If $A_{I I I}$ knows the secret key $S K_{I D^{*}} A_{I I I}$ can forge a legal signature. Let the event $E S K$ denote that $A_{I I I}$ knows the whole $S K_{I D^{*}}$ while $\overline{E S K}$ is the corresponding complement event. Let the event $E F S$ denote that $A_{I I I}$ can forge a legal signature. Hence, we have $A d v_{A I I I}=\operatorname{Pr}[E F S]$ that satisfies the following inequality

$$
\begin{aligned}
& A d v_{A I I I}=\operatorname{Pr}[E F S] \\
& =\operatorname{Pr}[E F S \wedge E S K]+\operatorname{Pr}[E F S \wedge \overline{E S K}] \\
& \leqq \operatorname{Pr}[E S K]+\operatorname{Pr}[E F S \wedge \overline{E S K}] .
\end{aligned}
$$

By the Signing leak query, $A_{I I I}$ derives at most $2 \lambda$ bits of $S K_{I D^{*}}$ By $\operatorname{Pr}[$ Case 1$] \leqq O\left(\left(q^{2} / p\right)\right.$ in Theorem 1 and Lemma 2 , we have $\operatorname{Pr}[E S K] \leqq O\left(\left(q^{2} / p\right)^{*} 2^{2 \lambda}\right)$. Finally, the event $\overline{E S K}$ is that $A_{I I I}$ can get fractional constituents of $\left(S K_{I D^{*}, k, 1}, S K_{I D^{*}, k, 2}\right)$ by $\Lambda f_{T K E, j}$ and $\Lambda h_{T K E, j}$. Since $A d v_{A I I-W} \leqq O\left(q^{2} / p\right)$ and $A_{I I I}$ can gain at most $2 \lambda$

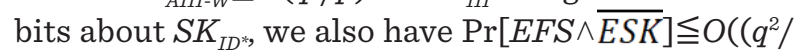
$p)^{*} 2^{2 \lambda}$ ). According to the discussions above, we have

$$
\begin{aligned}
& A d v_{A I I I}=\operatorname{Pr}[E F S] \leqq \operatorname{Pr}[E S K]+\operatorname{Pr}[E F S \wedge \overline{E S K}] \\
& \leqq O\left(\left(q^{2} / p\right)^{*} 2^{2 \lambda}\right) .
\end{aligned}
$$

By Corollary $1, A d v_{A I I}$ is negligible if $\lambda<\log p-\omega$ (loglog $p$ ). Q.E.D.

\section{Comparisons}

In this section, the comparisons between several previous RCLS and ORCLS schemes [7, 12, 21] and our
LR-ORCLS scheme are given. Firstly, let us define several computation notations. By the simulation experiences in [10], the corresponding computational costs (in millisecond) are given in Table 2. Note that we omit the computational costs of both the addition on $G$ and the multiplication on $G_{T}$ because they are small and negligible. For the simulation experiences in [10], the platform is equipped with a 3-GHz Pentium processor while running under a Microsoft window operation system. The security of the simulation results adopts 1024-bit RSA security level to measure the computational costs.

\section{Table 2}

Computational costs (in millisecond) of several operations

\begin{tabular}{c|l|c}
\hline Notations & \multicolumn{1}{|c|}{ Operations } & $\begin{array}{c}\text { Computational } \\
\text { costs }\end{array}$ \\
\hline$T_{b p}$ & a bilinear pairing $\hat{e}: G^{\prime} G^{\circledR} G_{T}$ & $20.01 \mathrm{~ms}$ \\
\hline$T_{s m}$ & a scalar multiplication on $G$ & $6.38 \mathrm{~ms}$ \\
\hline$T_{m p}$ & $\begin{array}{l}\text { a map-to-point hash } \\
\text { function on } G\end{array}$ & $3.04 \mathrm{~ms}$ \\
\hline$T_{c m}$ & $\begin{array}{l}\text { a scalar multiplication on } \\
\text { an elliptic curve group } G_{E C C}\end{array}$ & $0.83 \mathrm{~ms}$ \\
\hline
\end{tabular}

Table 3 demonstrates the comparisons between our LR-ORCLS scheme and several RCLS and ORCLS schemes $[7,12,21]$ in terms of signing cost (ms), verifying cost (ms), outsourced revocation, resisting side-channel attacks and overall leakage property. To provide leakage-resilient property (i.e., resisting side-channel attacks), our scheme requires some extra computation costs. It is obvious that the performance of our scheme is worse than the previously proposed RCLS and ORCLS schemes. Both Du et al.'s scheme and ours apply a CRS to offer outsourced revocation functionality to reduce the computational burden of the KGC. We emphasize that our scheme is the first LR-ORCLS scheme resistant against side-channel attacks while possessing overall unbounded leakage property. It is worth mentioning that the proposed scheme is not suited for unsuitable for some environments with resource-constrained devices (i.e. IoT devices) because it requires time-consuming bilinear pairing operations [33]. 
Table 3

Comparisons between our scheme and several previous RCLS or ORCLS schemes

\begin{tabular}{l|c|c|c|c} 
& $\begin{array}{c}\text { Sun } \text { et al.'s RCLS } \\
\text { scheme [21] }\end{array}$ & $\begin{array}{c}\text { Hung } \text { et al.'s RCLS } \\
\text { scheme [12] }\end{array}$ & $\begin{array}{c}\text { Du et al.'s ORCLS } \\
\text { scheme [7] }\end{array}$ & $\begin{array}{c}\text { Our LR-ORCLS } \\
\text { scheme }\end{array}$ \\
\hline Signing cost (ms) & $\begin{array}{c}2 T_{s m}+2 T_{m p} \\
(18.84 \mathrm{~ms})\end{array}$ & $\begin{array}{c}2 T_{s m}+2 T_{m p} \\
(18.84 \mathrm{~ms})\end{array}$ & $\begin{array}{c}T_{c m} \\
(0.83 \mathrm{~ms})\end{array}$ & $\begin{array}{c}5 T_{s m} \\
(31.9 \mathrm{~ms})\end{array}$ \\
\hline Verifying cost (ms) & $\begin{array}{l}3 T_{b p}+2 T_{m p} \\
(66.11 \mathrm{~ms})\end{array}$ & $\begin{array}{c}4 T_{b p}+T_{s m}+3 T_{m p} \\
(95.9 m s)\end{array}$ & $\begin{array}{c}5 T_{c m} \\
(4.15 \mathrm{~ms})\end{array}$ & $\begin{array}{c}4 T_{b p}+3 T_{s m} \\
(99.16 \mathrm{~ms})\end{array}$ \\
\hline Outsourced revocation & No & No & Yes & Yes \\
\hline Resisting side-channel attacks & No & No & No & Yes \\
\hline Overall leakage property & Not provided & Not provided & Not provided & Unbounded \\
\hline
\end{tabular}

\section{Conclusions and Future Work}

In this article, the first LR-ORCLS scheme has been proposed. As compared to previous RCLS and ORCLS schemes, our scheme has the following merits: (1) The revocation functionality is outsourced to the CRS to reduce the computational burden of the KGC; (2) It can resist side-channel attacks and permits adversaries to continually derive fractional constituents of private (or secret) keys; (3) It possesses the overall unbounded leakage property. Meanwhile, the novel adversary model was defined. By extending the adversary model of the ORCLS scheme, three kinds of leak queries are added, namely, Identity key leak query, Time update key leak query and Signing leak query. By three kinds of leak queries, adversaries are permitted to continually derive fractional constituents of the KGC's master secret key, the CRS's cloud secret key and a signer's secret key involved in the associated algorithms. In the GBG model, the security of the proposed scheme is shown to be existential unforgeable against Types I, II and III adversaries. By the comparisons mentioned in Table 3, indeed, our protocol still requires bilinear pairing operations and its performance is worse than the previous RCLS and ORCLS scheme. Hence, our protocol is unsuitable for some environments with resource-constrained devices (i.e. IoT devices). In the future, it is interesting to propose a lightweight LR-ORCLS protocol without requiring bilinear pairing operations.

\section{Acknowledgement}

This research was partially supported by Ministry of Science and Technology, Taiwan, under contract no. MOST108-2221-E-018-04-MY2.

\section{References}

1. Al-Riyami, S. S., Paterson, K. G. Certificateless Public Key Cryptography. In: ASIACRYPT'03, LNCS, 2894, Springer, Belin-Heidelberg, 2003, 452-473. https://doi. org/10.1007/978-3-540-40061-5_29

2. Alwen, J., Dodis, Y., Wichs, D. Leakage-Resilient Public-Key Cryptography in the Bounded-Retrieval Model.
In: CRYPTO`09, LNCS, 5677, Springer, Belin-Heidelberg, 2009, 36-54. https://doi.org/10.10077/978-3-64203356-8_3

3. Boneh, D., Boyen, X., Goh, E. J. Hierarchical Identity-Based Encryption with Constant Size Ciphertext. In: EUROCRYPT'05, LNCS, 3494, Spring- 
er, Belin-Heidelberg, 2005, 440-456. https://doi. org/10.1007/11426639_26

4. Boneh, D., Franklin, M. Identity-Based Encryption from the Weil Pairing. In: CRYPTO‘01, LNCS, 2139, Springer, Belin-Heidelberg, 2001, 213-229. https://doi. org/10.1007/3-540-44647-8_13

5. Brumley, D., Boneh, D. Remote Timing Attacks Are Practical. Computer Networks, 2005, 48(5), 701-716. https://doi.org/10.1016/j.comnet.2005.01.010

6. Dodis, Y., Haralambiev, K., Lopez-Alt, A., Wichs, D. Efficient Public-Key Cryptography in the Presence of Key Leakage. In: ASIACRYPT'10, LNCS, 647\%, Springer, Belin-Heidelberg, 2010, 613-631. https://doi. org/10.1007/978-3-642-17373-8_35

7. Du, H., Wen, Q., Zhang, S. A Provably-Secure Outsourced Revocable Certificateless Signature Scheme without Bilinear Pairings. IEEE Access, 2018, 6, 7384673855. https://doi.org/10.1109/ACCESS.2018.2880875

8. ElGamal, T. A Public Key Cryptosystem and a Signature Scheme Based on Discrete Logarithms. IEEE Transactions on Information Theory, 1985, 31(4), 469-472. https://doi.org/10.1109/TIT.1985.1057074

9. Galindo, D., Grobschadl, J., Liu, Z., Vadnala, P. K., Vivek, S. Implementation of a Leakage-Resilient ElGamal Key Encapsulation Mechanism. Journal of Cryptographic Engineering, 2016, 6(3), 229-238. https://doi. org/10.1007/s13389-016-0121-x

10. He, D., Chen, Y., Chen, J. An Efficient Certifcateless Proxy Signature Scheme without Pairing. Mathematical and Computer Modelling, 2013, 57, 2510-2518. https://doi.org/10.1016/j.mcm.2012.12.037

11. Housley, R., Polk, W., Ford, W., Solo, D. Internet X. 509 Public Key Infrastructure Certificate and Certificate Revocation List (CRL) Profile. IETF, RFC 3280, 2002. https://doi.org/10.17487/rfc3280

12. Hung, Y. H., Tseng, Y. M., Huang, S. S. A Revocable Certificateless Short Signature Scheme and Its Authentication Application. Informatica, 2016, 27(3), 549-572. https://doi.org/10.15388/Informatica.2016.99

13. Katz, J., Vaikuntanathan, V. Signature Schemes with Bounded Leakage Resilience. In: ASIACRYPT'09, LNCS, 5912, Springer, Belin-Heidelberg, 2009, 703720. https://doi.org/10.1007/978-3-642-10366-7_41

14. Kocher, P. Timing Attacks on Implementations of Diffie-Hellman, RSA, DSS, and Other Systems. In: CRYPTO‘96, LNCS, 1163, Springer, Belin-Heidelberg, 1996, 104-113. https://doi.org/10.10077/3-540-68697-5_9
15. Kocher, P., Jaffe, J., Jun, B. Differential Power Analysis. In: CRYPTO‘99, LNCS, 1666, Springer, Belin-Heidelberg, 1999, 388-397. https://doi.org/10.1007/3-54048405-1_25

16. Rivest, R. L., Shamir, A., Adleman, L. A Method for Obtaining Digital Signatures and Public-Key Cryptosystems. Communications of ACM, 1978, 21(2), 120-126. https://doi.org/10.1145/359340.359342

17. Scott, M. On the Efficient Implementation of Pairing-Based Protocols. In: Cryptography and Coding, LNCS, 7089, Springer, Belin-Heidelberg, 2011, 296308. https://doi.org/10.1007/978-3-642-25516-8_18

18. Shamir, A. Identity-Based Cryptosystems and Signature Schemes. In: CRYPTO‘84, LNCS, 196, Springer, Belin-Heidelberg, 1984, 47-53. https://doi.org/10.1007/3540-39568-7_5

19. Shen, L., Zhang, F., Sun, Y. Efficient Revocable Certificateless Encryption Secure in the Standard Model. Computer Journal, 2014, 57(4), 592-601. https://doi. org/10.1093/comjnl/bxt040

20. Shoup, V. Lower Bounds for Discrete Logarithms and Related Problems. In: EUROCRYPT'97, LNCS, 1233, Springer, Belin-Heidelberg, 1997, 256-266. https://doi. org/10.1007/3-540-69053-0_18

21. Sun, Y., Zhang, F., Shen, L. A Revocable Certificateless Signature Scheme. Journal of Computer, 2014, 9(8), 1843-1850. https://doi.org/10.4304/jcp.9.8.1843-1850

22. Tang, F., Li, H., Niu, Q., Liang, B. Efficient Leakage-Resilient Signature Schemes in the Generic Bilinear Group Model. In: Information Security Practice and Experience, LNCS, 8434, Springer, Belin-Heidelberg, 2014, 418-432. https://doi.org/10.1007/978-3-319-06320-1_31

23. Tsai, T. T., Tseng, Y. M. Revocable Certificateless Public Key Encryption. IEEE Systems Journal, 2015, 9(3), 824-833. https://doi.org/10.1109/JSYST.2013.2289271

24. Tsai, T. T., Huang, S. S., Tseng, Y. M. Secure Certificateless Signature with Revocation in the Standard Model. Mathematical Problems in Engineering, 2014, Article ID 728591. https://doi.org/10.1155/2014/728591

25. Tseng, Y. M., Tsai, T. T. Efficient Revocable ID-Based Encryption with a Public Channel. Computer Journal, 2012, 55(4), 475-486. https://doi.org/10.1093/comjnl/ bxr098

26. Wu, J. D., Tseng, Y. M., Huang, S. S. Leakage-Resilient IDBased Signature Scheme in the Generic Bilinear Group Model. Security and Communication Networks, 2016, 9(17), 3987-4001. https://doi.org/10.1002/sec.1580 
27. Wu, J. D., Tseng, Y. M., Huang, S. S. Leakage-Resilient Certificateless Signature under Continual Leakage Model. Information Technology and Control, 2018, 47(2), 363-386. https://doi.org/10.5755/j01. itc. 47.2 .17847

28. Wu, J. D., Tseng, Y. M., Huang, S. S. Efficient Leakage-Resilient Authenticated Key Agreement Protocol in the Continual Leakage eCK Model. IEEE Access, 2018, 6(1), 17130-17142. https://doi.org/10.1109/ACCESS.2018.2799298

29. Wu, J. D., Tseng, Y. M., Huang, S. S. An Identity-Based Authenticated Key Exchange Protocol Resilient to Continuous Key Leakage. IEEE Systems Journal, 2019, 13(4), 3968-3979. https://doi.org/10.1109/ JSYST.2019.2896132

30. Wu, J. D., Tseng, Y. M., Huang, S. S., Tsai, T. T. Leakage-Resilient Certificate-Based Signature Resistant to
Side-Channel Attacks. IEEE Access, 2019, 7(1), 1904119053. https://doi.org/10.1109/ACCESS.2019.2896773

31. Xiong, H., Yuen, T. H., Zhang, C., Yiu, S. M., He, Y. J. Leakage-Resilient Certificateless Public Key Encryption. In: Proceedings of the first ACM workshop on Asia public-key cryptography, 2013, 13-22. https://doi. org/10.1145/2484389.2484394

32. Zhou, Y., Yang, B., Zhang, W. Provably Secure and Efficient Leakage-Resilient Certificateless Signcryption Scheme without Bilinear Pairing. Discrete Applied Mathematics, 2016, 204, 185-202. https://doi. org/10.1016/j.dam.2015.10.018

33. Zhou, L., Su, C., Yeh, K. A Lightweight Cryptographic Protocol with Certificateless Signature for the Internet of Things. ACM Transactions on Embedded Computing Systems, 2019, 18(3), Article 28. https://doi. org/10.1145/3301306 